\title{
A Topos Foundation for Theories of Physics: III. The Representation of Physical Quantities With Arrows $\breve{\delta}^{o}(A): \underline{\Sigma} \rightarrow \underline{\mathbb{R}^{\succeq}}$
}

\author{
A. Döring 1 \\ and \\ C.J. Isham 2 \\ The Blackett Laboratory \\ Imperial College of Science, Technology \& Medicine \\ South Kensington \\ London SW7 2BZ
}

6 March, 2007

\begin{abstract}
This paper is the third in a series whose goal is to develop a fundamentally new way of viewing theories of physics. Our basic contention is that constructing a theory of physics is equivalent to finding a representation in a topos of a certain formal language that is attached to the system.

In paper II, we studied the topos representations of the propositional language $\mathcal{P} \mathcal{L}(S)$ for the case of quantum theory, and in the present paper we do the same thing for the, more extensive, local language $\mathcal{L}(S)$. One of the main achievements is to find a topos representation for self-adjoint operators. This involves showing that, for any physical quantity $A$, there is an arrow $\delta^{o}(A): \underline{\Sigma} \rightarrow \underline{\mathbb{R}} \succeq$, where $\underline{\mathbb{R}^{\succeq}}$ is the quantity-value object for this theory. The construction of $\check{\delta}^{o}(\hat{A})$ is an extension of the daseinisation of projection operators that was discussed in paper II.

The object $\underline{\mathbb{R}} \succeq$ is a monoid-object only in the topos, $\tau_{\phi}=\operatorname{Sets}^{\mathcal{V}(\mathcal{H})^{\mathrm{op}}}$, of the theory, and to enhance the applicability of the formalism, we apply to $\underline{\mathbb{R}} \succeq$ a topos analogue of the Grothendieck extension of a monoid to a group. The resulting object, $k(\underline{\mathbb{R}} \succeq)$, is an abelian group-object in $\tau_{\phi}$. We also discuss another candidate, $\underline{\mathbb{R} \leftrightarrow}$, for the quantity-value object. In this presheaf, both inner and outer daseinisation are used in a symmetric way.

Finally, there is a brief discussion of the role of unitary operators in the quantum topos scheme.
\end{abstract}

\footnotetext{
${ }^{1}$ email: a.doering@imperial.ac.uk

${ }^{2}$ email: c.isham@imperial.ac.uk
} 


\section{Introduction}

This is the third in a series of papers whose aim is to construct a general framework within which theories of physics can be developed in a topos other than that of sets. A theme that runs throughout this work is the idea that any 'non-classical' theory, for example, quantum theory, can be presented in a way that 'looks like' classical physics, except that the topos is generally not the topos of sets. This approach provides a new set of tools with which to construct theories of physics. At the conceptual level, the analogue with classical physics leads to the scheme being 'neo-realist'.

In paper I, we introduced the idea of associating a formal language with each physical system $S$ [2]. Constructing a theory of physics is then equivalent to finding a representation of this language in a topos. Two different kinds of language are discussed in paper I: a simple propositional language $\mathcal{P} \mathcal{L}(S)$; and a more sophisticated, higherorder language (a 'local' language) $\mathcal{L}(S)$.

The language $\mathcal{P} \mathcal{L}(S)$ provides a deductive system (using intuitionistic logic) and hence provides a way of making statements about the system $S$. However, a purely propositional language is limited in scope: at the very least, one would like to have a 'first-order' language, so that the phrases 'for all' and 'there exists' can be used.

But such a language is still rudimentary in so far as many features of a physical theory would lie outside its scope, and are introduced only when constructing a representation. For example, in classical mechanics, the entities that lie outside the language are (i) the state space $\mathcal{S}$; (ii) the choice of $\mathbb{R}$ as the set in which physical quantities take their values; (iii) the specific subset $\Delta \subseteq \mathbb{R}$ that is used in the proposition " $A \varepsilon \Delta$ " and (iv) the real-valued functions on $\mathcal{S}$ that represent physical quantities.

For this reason, the next step in paper I was to assign to each physical system $S$, a more powerful, typed language, $\mathcal{L}(S)$. Our general scheme can then be understood as the task of finding representations of $\mathcal{L}(S)$ in various topoi. The language $\mathcal{L}(S)$ has two 'ground-type' symbols, $\Sigma$ and $\mathcal{R}$, and a set of 'function symbols', written rather suggestively as the string of characters ' $A: \Sigma \rightarrow \mathcal{R}$ '. These are the linguistic precursors of, respectively, the state object, the quantity-value object, and the arrows between them that represent physical quantities. A symbol $\tilde{\Delta}$ can be introduced as a variable of type $P \mathcal{R}$. By these means, the entities that lie outside the propositional language $\mathcal{P} \mathcal{L}(S)$ are all brought 'inside' the local language $\mathcal{L}(S)$.

The second paper in the series dealt with the topos representation of the propositional language $\mathcal{P} \mathcal{L}(S)$ in quantum theory [3]. In a sense this paper is a side-line to our main programme which is concerned with the local language $\mathcal{L}(S)$. In fact, logically speaking, we could have omitted our study of the representations of $\mathcal{P} \mathcal{L}(S)$. However, the $\mathcal{P} \mathcal{L}(S)$ material links most closely with the original work on the use of topos ideas in quantum theory. Also, as we will see, the vital concept of 'daseinisation' of propositions has a natural extension to physical quantities in general, and this plays a key role in the remaining papers in the series. 
In the present paper, we return to the language $\mathcal{L}(S)$ and seek a topos representation, $\phi$, for quantum theory. As discussed in paper II, the topos of the $\mathcal{P} \mathcal{L}(S)$ representation is $\operatorname{Sets}^{\mathcal{V}(\mathcal{H})^{\mathrm{op}}}$ : the topos of presheaves over the category, $\mathcal{V}(\mathcal{H})$, of unital, abelian subalgebras of the algebra $\mathcal{B}(\mathcal{H})$ of all bounded operators on the quantum Hilbert space $\mathcal{H}$. We shall use the same topos for the $\mathcal{L}(S)$-representation, with the spectral presheaf, $\underline{\Sigma}$, being identified as the $\operatorname{Sets}^{\mathcal{V}(\mathcal{H})^{\mathrm{op}}}$-representative, $\Sigma_{\phi}$, of the ground-type symbol $\Sigma$. Thus $\underline{\Sigma}$ is the state object, and, therefore, propositions are represented by sub-objects of $\underline{\Sigma}$; just as, in classical physics, a proposition about the system is represented by a subset of the classical state space.

The steps in finding the representation of $\mathcal{L}(S)$ are first to identify the quantityvalue object, $\mathcal{R}_{\phi}$; and then to show how to represent a physical quantity, $A$, by an arrow $\breve{\delta}(A): \underline{\Sigma} \rightarrow \mathcal{R}_{\phi}$. Both problems are solved in the present paper.

The plan of the paper is as follows. We start in Section 2 by defining the inner and outer daseinisations of an arbitrary self-adjoint operator $\hat{A} \in \mathcal{B}(\mathcal{H})$. These constructions utilise the daseinisations of projection operators that were discussed in paper II. The result is two quantities, $\delta^{i}(\hat{A})$ and $\delta^{\circ}(\hat{A})$, that can be identified as global elements of two new presheaves: the 'inner-' and the 'outer de Groote presheaf', respectively.

In Section 3, for each stage $V \in \mathrm{Ob}(\mathcal{V}(\mathcal{H}))$, we take the Gel'fand transforms of $\delta^{o}(\hat{A})_{V}$, and show how the target spaces of these transforms fit together to give a new presheaf, $\operatorname{sp}(\hat{A})^{\succeq}$, which is a topos extension of the $\operatorname{spectrum,} \operatorname{sp}(\hat{A})$, of $\hat{A}$. For any self-adjoint operator $\hat{A}$, the target presheaf $\operatorname{sp}(\hat{A}) \succeq$ can be embedded as a sub-object of a single presheaf $\underline{\mathbb{R}} \succeq$. In this way, $\underline{\mathbb{R}} \succeq$ is provisionally identified as the quantityvalue presheaf for quantum theory in the topos $\operatorname{Sets}^{\mathcal{V}(\mathcal{H})^{\text {op }}}$. There is also an isomorphic presheaf, $\underline{\mathbb{R}} \preceq$, that is constructed using inner daseinisation; and a third presheaf, $\underline{\mathbb{R}} \leftrightarrow$, that combines both inner and outer daseinisation. This is the presheaf that gives the most natural physical interpretation of the arrows $\breve{\delta}(A): \underline{\Sigma} \rightarrow \underline{\mathbb{R}} \leftrightarrow$.

However, algebraically speaking, $\underline{\mathbb{R}^{\succeq}}, \underline{\mathbb{R}} \preceq$, and $\underline{\mathbb{R}} \leftrightarrow$ are only additive monoidobjects in the topos, whereas the set of real numbers, $\mathbb{R}$, employed in standard physics, is an abelian group (in fact, even an abelian ring). This limits what can be done with these presheaves and motivates us to apply a standard trick in algebraic topology. This is the 'Grothendieck completion' of a monoid to give a group, which we adapt to produce a presheaf, $k\left(\underline{\mathbb{R}^{\succeq}}\right)$, that extends the monoid-object $\underline{\mathbb{R}^{\succeq}}$ to a full group-object in the topos $\operatorname{Sets}^{\mathcal{V}(\mathcal{H})^{\text {op }}}$. We show that the square can be taken of certain elements in $k\left(\underline{\mathbb{R}^{\succeq}}\right)$, which enables us to define a kind of dispersion for daseinised self-adjoint operators. We also show that the presheaf $k\left(\underline{\mathbb{R}^{\succeq}}\right)$ is closely related to $\underline{\mathbb{R}^{\leftrightarrow}}$.

Finally, in Section 5, we recall the role of unitary operators in standard quantum theory, and show how each such operator, $\hat{U}$, can be made to act as a functor from the

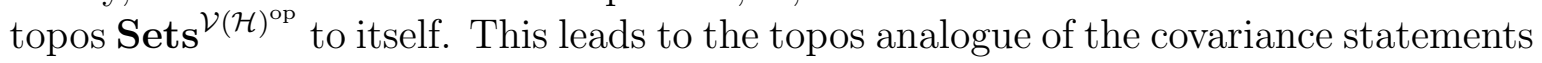
that are associated with unitary operators in quantum theory. 


\section{The de Groote Presheaves of Physical Quantities}

\subsection{Background Remarks}

Our task is to consider the representation of the local language, $\mathcal{L}(S)$, in the case of quantum theory. We assume that the relevant topos is the same as that used for the propositional language $\mathcal{P} \mathcal{L}(S)$, i.e., $\operatorname{Sets}^{\mathcal{V}(\mathcal{H})^{\mathrm{op}}}$, but the emphasis is very different.

From a physics perspective, the key symbols in $\mathcal{L}(S)$ are the ground-type symbols, $\Sigma$ and $\mathcal{R}$ - the linguistic precursors of the state object and the quantity-value object respectively - and the function symbols $A: \Sigma \rightarrow \mathcal{R}$, which are the precursors of physical quantities. In the quantum-theory representation, $\phi$, of $\mathcal{L}(S)$, the representation, $\Sigma_{\phi}$, of $\Sigma$ is defined to be the spectral presheaf $\underline{\Sigma}$ in the topos $\operatorname{Sets}^{\mathcal{V}(\mathcal{H})^{\mathrm{op}}}$.

The critical question is to find the object, $\mathcal{R}_{\phi}$ (provisionally denoted as a presheaf $\underline{\mathcal{R}})$, in $\operatorname{Sets}^{\mathcal{V}(\mathcal{H})^{\text {op }}}$ that represents $\mathcal{R}$, and is hence the quantity-value object. One might think that $\underline{\mathcal{R}}$ is the real-number object in the topos $\operatorname{Sets}^{\mathcal{V}(\mathcal{H})^{\text {op }}}$, but that is wrong, and the right answer cannot just be guessed. In fact, the correct choice for $\underline{\mathcal{R}}$ is found indirectly by considering a related question: namely, how to represent each function symbol $A: \Sigma \rightarrow \mathcal{R}$, with a concrete arrow $A_{\phi}: \Sigma_{\phi} \rightarrow \mathcal{R}_{\phi}$ in $\operatorname{Sets}^{\mathcal{V}(\mathcal{H})^{\text {op }}}$, i.e., with a natural transformation $\breve{A}: \underline{\Sigma} \rightarrow \underline{\mathcal{R}}$ between the presheaves $\underline{\Sigma}$ and $\underline{\mathcal{R}}$.

\subsection{The Outer and Inner Presheaves}

The daseinisation operations on projection operators were introduced by de Groote [6], and exploited by us in [3]. They are defined as follows.

Definition 2.1 If $\hat{P}$ is a projection operator, and $V \in \mathrm{Ob}(\mathcal{V}(\mathcal{H}))$ is any context/stage, we define:

1. The 'outer daseinisation' operation is

$$
\delta^{o}(\hat{P})_{V}:=\bigwedge\{\hat{Q} \in \mathcal{P}(V) \mid \hat{Q} \succeq \hat{P}\}
$$

where ' $\succeq$ ' denotes the usual ordering of projection operators, and where $\mathcal{P}(V)$ is the set of all projection operators in $V$.

2. Similarly, the 'inner daseinisation' operation is defined in the context $V$ as

$$
\delta^{i}(\hat{P})_{V}:=\bigvee\{\hat{Q} \in \mathcal{P}(V) \mid \hat{Q} \preceq \hat{P}\}
$$

Thus $\delta^{o}(\hat{P})_{V}$ is the best approximation to $\hat{P}$ in $V$ from 'above', being the smallest projection in $V$ that is larger than or equal to $\hat{P}$. Similarly, $\delta^{i}(\hat{P})_{V}$ is the best approximation to $\hat{P}$ from 'below', being the largest projection in $V$ that is smaller than or equal to $\hat{P}$. 
Daseinisation was used in paper II in the construction of the 'outer' and 'inner' presheaves $\underline{O}$ and $\underline{I}$, which are defined as follows:

Definition 2.2 The outer presheaf, $\underline{O}$, is defined over the category $\mathcal{V}(\mathcal{H})$ by:

(i) On objects $V \in \operatorname{Ob}(\mathcal{V}(\mathcal{H})): \underline{O}_{V}:=\mathcal{P}(V)$.

(ii) On morphisms $i_{V^{\prime} V}: V^{\prime} \subseteq V$ : Define $\underline{O}\left(i_{V^{\prime} V}\right): \underline{O}_{V} \rightarrow \underline{O}_{V^{\prime}}$ by $\underline{O}\left(i_{V^{\prime} V}\right)(\hat{\alpha}):=$ $\delta^{o}(\hat{\alpha})_{V^{\prime}}$ for all $\hat{\alpha} \in \mathcal{P}(V)$.

Evidently, the map $V \mapsto \delta^{o}(\hat{P})_{V}$ defines a global element, $\delta^{o}(\hat{P})$, of the presheaf $\underline{O}$.

Definition 2.3 The inner presheaf, $\underline{I}$, is defined over the category $\mathcal{V}(\mathcal{H})$ by:

(i) On objects $V \in \operatorname{Ob}(\mathcal{V}(\mathcal{H})): \underline{I}_{V}:=\mathcal{P}(V)$.

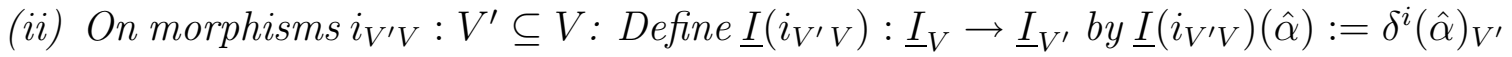
for all $\hat{\alpha} \in \mathcal{P}(V)$.

Evidently, the map $V \mapsto \delta^{i}(\hat{P})_{V}$ defines a global element, $\delta^{i}(\hat{P})$, of the inner presheaf $\underline{I}$.

In paper II, we showed that the outer presheaf is a sub-object of the power object $P_{\mathrm{cl}} \underline{\Sigma}$ (in the category $\operatorname{Sets}^{\mathcal{V}(\mathcal{H})^{\mathrm{op}}}$ ), and hence that the global element $\delta^{o}(\hat{P})$ of $\underline{O}$ determines a (clopen) sub-object of the spectral presheaf $\underline{\Sigma}$. By these means, the quantum logic of the lattice $\mathcal{P}(\mathcal{H})$ is mapped into the Heyting algebra of the set, $\operatorname{Sub}_{\mathrm{cl}}(\underline{\Sigma})$, of clopen sub-objects of $\underline{\Sigma}$.

Our task in the present paper is to perform the second stage of the quantum programme: namely (i) identify the quantity-value presheaf, $\underline{\mathcal{R}}$; and (ii) show that any physical quantity can be represented by an arrow from $\underline{\Sigma}$ to $\underline{\mathcal{R}}$.

\subsection{The Daseinisation of an Arbitrary Self-Adjoint Operator}

\subsubsection{Spectral Families and Spectral Order}

We now want to extend the daseinisation operations from projections to arbitrary (bounded) self-adjoint operators. To this end, consider first a bounded, self-adjoint operator, $\hat{A}$, whose spectrum is purely discrete. Then the spectral theorem can be used to write $\hat{A}=\sum_{i=1}^{\infty} a_{i} \hat{P}_{i}$ where $a_{1}, a_{2}, \ldots$ are the eigenvalues of $\hat{A}$, and $\hat{P}_{1}, \hat{P}_{2}, \ldots$ are the spectral projection operators onto the corresponding eigenspaces.

A construction that comes immediately to mind is to use the daseinisation operation on projections to define

$$
\delta^{o}(\hat{A})_{V}:=\sum_{i=1}^{\infty} a_{i} \delta^{o}\left(\hat{P}_{i}\right)_{V}
$$


for each stage $V$. However, this procedure is rather unnatural. For one thing, the projections, $\hat{P}_{i}, i=1,2, \ldots$ form a complete orthonormal set:

$$
\begin{aligned}
\sum_{i=1}^{\infty} \hat{P}_{i} & =\hat{1}, \\
\hat{P}_{i} \hat{P}_{j} & =\delta_{i j} \hat{P}_{i},
\end{aligned}
$$

whereas, in general, the collection of daseinised projections, $\delta^{o}\left(\hat{P}_{i}\right)_{V}, 1=1,2, \ldots$ will not satisfy either of these conditions. In addition, it is hard to see how the expression $\delta^{o}(\hat{A})_{V}:=\sum_{i=1}^{\infty} a_{i} \delta^{o}\left(\hat{P}_{i}\right)_{V}$ can be generalised to operators, $\hat{A}$, with a continuous spectrum.

The answer to this conundrum lies in the work of de Groote. He realised that although it is not useful to daseinise the spectral projections of an operator $\hat{A}$, it is possible to daseinise the spectral family of $\hat{A}[\underline{6}$.

Spectral families. We first recall that a spectral family is a family of projection operators $\hat{E}_{\lambda}, \lambda \in \mathbb{R}$, with the following properties:

1. If $\lambda_{2} \leq \lambda_{1}$ then $\hat{E}_{\lambda_{2}} \preceq \hat{E}_{\lambda_{1}}$.

2. The net $\lambda \mapsto \hat{E}_{\lambda}$ of projection operators in the lattice $\mathcal{P}(\mathcal{H})$ is bounded above by $\hat{1}$, and below by $\hat{0}$. In fact,

$$
\begin{aligned}
\lim _{\lambda \rightarrow \infty} \hat{E}_{\lambda} & =\hat{1} \\
\lim _{\lambda \rightarrow-\infty} \hat{E}_{\lambda} & =\hat{0} .
\end{aligned}
$$

3. The map $\lambda \mapsto \hat{E}_{\lambda}$ is right continuous:3

$$
\bigwedge_{\epsilon \downarrow 0} \hat{E}_{\lambda+\epsilon}=\hat{E}_{\lambda}
$$

for all $\lambda \in \mathbb{R}$.

The spectral theorem asserts that for any self-adjoint operator $\hat{A}$, there exists a spectral family, $\lambda \mapsto \hat{E}_{\lambda}^{A}$, such that

$$
\hat{A}=\int_{\mathbb{R}} \lambda d \hat{E}_{\lambda}^{A}
$$

We are only concerned with bounded operators, and so the (weak Stieljes) integral in (2.9) is really over the bounded spectrum of $\hat{A}$ which, of course, is a compact subset of $\mathbb{R}$. Conversely, given a bounded spectral family $\left\{\hat{E}_{\lambda}\right\}_{\lambda \in \mathbb{R}}$, 4 there is a bounded self-adjoint operator $\hat{A}$ such that $\hat{A}=\int_{\mathbb{R}} \lambda d \hat{E}_{\lambda}$.

\footnotetext{
${ }^{3}$ It is a matter of convention whether one chooses right-continuous or left-continuous.

${ }^{4}$ I.e., there are $a, b \in \mathbb{R}$ such that $\hat{E}_{\lambda}=\hat{0}$ for all $\lambda \leq a$ and $\hat{E}_{\lambda}=\hat{1}$ for all $\lambda \geq b$.
} 
The spectral order. A key element for our work is the so-called spectral order that was introduced in [17] 5 It is defined as follows. Let $\hat{A}$ and $\hat{B}$ be (bounded) self-adjoint operators with spectral families $\left\{\hat{E}_{\lambda}^{A}\right\}_{\lambda \in \mathbb{R}}$ and $\left\{\hat{E}_{\lambda}^{B}\right\}_{\lambda \in \mathbb{R}}$, respectively. Then define:

$$
\hat{A} \preceq_{s} \hat{B} \text { if and only if } \hat{E}_{\lambda}^{B} \preceq \hat{E}_{\lambda}^{A} \text { for all } \lambda \in \mathbb{R} \text {. }
$$

It is easy to see that $(2.10)$ defines a genuine partial ordering on $\mathcal{B}(\mathcal{H})_{\text {sa }}$ (the self-adjoint operators in $\mathcal{B}(\mathcal{H})$ ). In fact, $\mathcal{B}(\mathcal{H})_{\text {sa }}$ is a 'boundedly complete' lattice with respect to the spectral order, i.e., each bounded set $S$ of self-adjoint operators has a minimum $\bigwedge S \in \mathcal{B}(\mathcal{H})_{\text {sa }}$ and a maximum $\bigvee S \in \mathcal{B}(\mathcal{H})_{\text {sa }}$ with respect to this order.

If $\hat{P}, \hat{Q}$ are projections, then

$$
\hat{P} \preceq_{s} \hat{Q} \text { if and only if } \hat{P} \preceq \hat{Q},
$$

so the spectral order coincides with the usual partial order on $\mathcal{P}(\mathcal{H})$. To ensure this, the 'reverse' relation in (2.10) is necessary, since the spectral family of a projection $\hat{P}$ is given by

$$
E_{\lambda}^{\hat{P}}= \begin{cases}\hat{0} & \text { if } \lambda<0 \\ \hat{1}-\hat{P} & \text { if } 0 \leq \lambda<1 \\ \hat{1} & \text { if } \lambda \geq 1\end{cases}
$$

If $\hat{A}, \hat{B}$ are self-adjoint operators such that (i) either $\hat{A}$ or $\hat{B}$ is a projection, or (ii) $[\hat{A}, \hat{B}]=\hat{0}$, then $\hat{A} \preceq{ }_{s} \hat{B}$ if and only if $\hat{A} \preceq \hat{B}$. Here ' $\preceq$ ' denotes the usual ordering on $\mathcal{B}(\mathcal{H})_{\mathrm{sa}} 6$

Moreover, if $\hat{A}, \hat{B}$ are arbitrary self-adjoint operators, then $\hat{A} \preceq{ }_{s} \hat{B}$ implies $\hat{A} \preceq \hat{B}$, but not vice versa in general. Thus the spectral order is a partial order on $\mathcal{B}(\mathcal{H})_{\mathrm{sa}}$ that is coarser than the usual one.

\subsubsection{Daseinisation of Self-Adjoint Operators.}

De Groote's crucial observation was the following. Let $\lambda \mapsto \hat{E}_{\lambda}$ be a spectral family in $\mathcal{P}(\mathcal{H})$ (or, equivalently, a self-adjoint operator $\hat{A}$ ). Then, for each stage $V$, the following maps:

$$
\begin{aligned}
\lambda & \mapsto \bigwedge_{\mu>\lambda} \delta^{o}\left(\hat{E}_{\mu}\right)_{V} \\
\lambda & \mapsto \delta^{i}\left(\hat{E}_{\lambda}\right)_{V}
\end{aligned}
$$

also define spectral families, 7 These spectral families lie in $\mathcal{P}(V)$ and hence, by the spectral theorem, define self-adjoint operators in $V$. This leads to the definition of the two daseinisations of an arbitrary self-adjoint operator:

\footnotetext{
${ }^{5}$ The spectral order was later reinvented by de Groote, see [5].

${ }^{6}$ The 'usual' ordering is $\hat{A} \preceq \hat{B}$ if $\langle\psi|\hat{A}| \psi\rangle \leq\langle\psi|\hat{B}| \psi\rangle$ for all vectors $|\psi\rangle \in \mathcal{H}$.

${ }^{7}$ The reason (2.13) and (2.14) have a different form is that $\lambda \mapsto \delta^{i}\left(\hat{E}_{\lambda}\right)_{V}$ is right continuous whereas $\lambda \mapsto \delta^{o}\left(\hat{E}_{\lambda}\right)_{V}$ is not. On the other hand, the family $\lambda \mapsto \bigwedge_{\mu>\lambda} \delta^{o}\left(\hat{E}_{\mu}\right)_{V}$ is right continuous.
} 
Definition 2.4 Let $\hat{A}$ be an arbitrary self-adjoint operator. Then the outer and inner daseinisations of $\hat{A}$ are defined at each stage $V$ as:

$$
\begin{aligned}
\delta^{o}(\hat{A})_{V} & :=\int_{\mathbb{R}} \lambda d\left(\delta_{V}^{i}\left(\hat{E}_{\lambda}^{A}\right)\right), \\
\delta^{i}(\hat{A})_{V} & :=\int_{\mathbb{R}} \lambda d\left(\bigwedge_{\mu>\lambda} \delta_{V}^{o}\left(\hat{E}_{\mu}^{A}\right)\right),
\end{aligned}
$$

respectively.

Note that for all $\lambda \in \mathbb{R}$, and for all stages $V$, we have

$$
\delta^{i}\left(\hat{E}_{\lambda}\right)_{V} \preceq \bigwedge_{\mu>\lambda} \delta^{o}\left(\hat{E}_{\mu}\right)_{V}
$$

and hence, for all $V$,

$$
\delta^{i}(\hat{A})_{V} \preceq_{s} \delta^{o}(\hat{A})_{V}
$$

This explains why the ' $i$ ' and ' $o$ ' superscripts in (2.15 2.16) are defined the way round that they are.

Both outer daseinisation (2.15) and inner daseinisation (2.16) can be used to 'adapt' a self-adjoint operator $\hat{A}$ to contexts $V \in \mathrm{Ob}(\mathcal{V}(\mathcal{H}))$ that do not contain $\hat{A}$. (On the other hand, if $\hat{A} \in V$, then $\delta^{o}(\hat{A})_{V}=\delta^{i}(\hat{A})_{V}=\hat{A}$.)

\subsubsection{Properties of daseinisation.}

We will now list some useful properties of daseinisation.

1. It is clear that the outer, and inner, daseinisation operations can be extended to situations where the self-adjoint operator $\hat{A}$ does not belong to $\mathcal{B}(\mathcal{H})_{\text {sa }}$, or where $V$ is not an abelian subalgebra of $\mathcal{B}(\mathcal{H})$. Specifically, let $\mathcal{N}$ be an arbitrary, unital von Neumann algebra, and let $\mathcal{S} \subset \mathcal{N}$ be a proper unital von Neumann subalgebra. Then outer and inner daseinisation can be defined as the mappings

$$
\begin{aligned}
\delta^{o}: \mathcal{N}_{\mathrm{sa}} & \rightarrow \mathcal{S}_{\mathrm{sa}} \\
\hat{A} & \mapsto \int_{\mathbb{R}} \lambda d\left(\delta_{\mathcal{S}}^{i}\left(\hat{E}_{\lambda}^{A}\right)\right), \\
\delta^{i}: \mathcal{N}_{\mathrm{sa}} & \rightarrow \mathcal{S}_{\mathrm{sa}} \\
\hat{A} & \mapsto \int_{\mathbb{R}} \lambda d\left(\bigwedge_{\mu>\lambda} \delta_{\mathcal{S}}^{o}\left(\hat{E}_{\mu}^{A}\right)\right) .
\end{aligned}
$$

A particular case is $\mathcal{N}=V$ and $\mathcal{S}=V^{\prime}$ for two contexts $V, V^{\prime}$ such that $V^{\prime} \subset V$. Hence, a self-adjoint operator can be restricted from one context to a sub-context.

For the moment, we will let $\mathcal{N}$ be an arbitrary unital von Neumann algebra, with $\mathcal{S} \subset \mathcal{N}$ 
2. By construction,

$$
\delta^{o}(\hat{A})_{\mathcal{S}}=\bigwedge\left\{\hat{B} \in \mathcal{S}_{\mathrm{sa}} \mid \hat{B} \succeq_{s} \hat{A}\right\}
$$

where the minimum is taken with respect to the spectral order; i.e., $\delta^{o}(\hat{A})_{\mathcal{S}}$ is the smallest self-adjoint operator in $\mathcal{S}$ that is spectrally larger than (or equal to) $\hat{A}$. This implies $\delta^{o}(\hat{A})_{\mathcal{S}} \succeq \hat{A}$ in the usual order. Likewise,

$$
\delta^{i}(\hat{A})_{\mathcal{S}}=\bigvee\left\{\hat{B} \in \mathcal{S} \mid \hat{B} \preceq_{s} \hat{A}\right\}
$$

so $\delta^{i}(\hat{A})_{\mathcal{S}}$ is the largest self-adjoint operator in $\mathcal{S}$ spectrally smaller than (or equal to) $\hat{A}$, which implies $\delta^{i}(\hat{A})_{\mathcal{S}} \preceq \hat{A}$.

3. In general, neither $\delta^{o}(\hat{A})_{\mathcal{S}}$ nor $\delta^{i}(\hat{A})_{\mathcal{S}}$ can be written as Borel functions of the operator $\hat{A}$, since daseinisation changes the elements of the spectral family, while a function merely 'shuffles them around'.

4. Let $\hat{A} \in \mathcal{N}$ be self-adjoint. The spectrum, $\operatorname{sp}(\hat{A})$, consists of all $\lambda \in \mathbb{R}$ such that the spectral family $\left\{\hat{E}_{\lambda}^{A}\right\}_{\lambda \in \mathbb{R}}$ is non-constant on any neighbourhood of $\lambda$. By definition, outer daseinisation of $\hat{A}$ acts on the spectral family of $\hat{A}$ by sending $\hat{E}_{\lambda}^{A}$ to $\hat{E}_{\lambda}^{\delta^{o}(\hat{A})_{\mathcal{S}}}=\delta^{i}\left(\hat{E}_{\lambda}^{A}\right)_{\mathcal{S}}$. If $\left\{\hat{E}_{\lambda}^{A}\right\}_{\lambda \in \mathbb{R}}$ is constant on some neighbourhood of $\lambda$, then the spectral family $\left\{\hat{E}_{\lambda}^{\delta^{o}(\hat{A})_{\mathcal{S}}}\right\}_{\lambda \in \mathbb{R}}$ of $\delta^{o}(\hat{A})_{\mathcal{S}}$ is also constant on this neighbourhood. This shows that

$$
\operatorname{sp}\left(\delta^{o}(\hat{A})_{\mathcal{S}}\right) \subseteq \operatorname{sp}(\hat{A})
$$

for all self-adjoint operators $\hat{A} \in \mathcal{N}_{\text {sa }}$ and all von Neumann subalgebras $\mathcal{S}$. Analogous arguments apply to inner daseinisation.

Heuristically, this result implies that the spectrum of the operator $\delta^{o}(\hat{A})_{\mathcal{S}}$ is more degenerate than that of $\hat{A}$; i.e., the effect of daseinisation is to 'collapse' eigenvalues.

5. Outer and inner daseinisation are both non-linear mappings. We will show this for projections explicitly. For example, let $\hat{Q}:=\hat{1}-\hat{P}$. Then $\delta^{o}(\hat{Q}+\hat{P})_{\mathcal{S}}=\delta^{o}(\hat{1})_{\mathcal{S}}=\hat{1}$, while $\delta^{o}(\hat{1}-\hat{P})_{\mathcal{S}} \succ \hat{1}-\hat{P}$ and $\delta^{o}(\hat{P})_{\mathcal{S}} \succ \hat{P}$ in general, so $\delta^{o}(\hat{1}-\hat{P})_{\mathcal{S}}+\delta^{o}(\hat{P})_{\mathcal{S}}$ is the sum of two non-orthogonal projections in general (and hence not equal to $\hat{1}$ ). For inner daseinisation, we have $\delta^{i}(\hat{1}-\hat{P})_{\mathcal{S}} \prec \hat{1}-\hat{P}$ and $\delta^{i}(\hat{P})_{\mathcal{S}} \prec \hat{P}$ in general, so $\delta^{i}(\hat{1}-\hat{P})_{\mathcal{S}}+\delta^{i}(\hat{P})_{\mathcal{S}} \prec \hat{1}=\delta^{i}(\hat{1}-\hat{P}+\hat{P})_{\mathcal{S}}$ in general.

6. If $a \geq 0$, then $\delta^{o}(a \hat{A})_{\mathcal{S}}=a \delta^{o}(\hat{A})_{\mathcal{S}}$ and $\delta^{i}(a \hat{A})_{\mathcal{S}}=a \delta^{i}(\hat{A})_{\mathcal{S}}$. If $a<0$, then $\delta^{o}(a \hat{A})_{\mathcal{S}}=a \delta^{i}(\hat{A})_{\mathcal{S}}$ and $\delta^{i}(a \hat{A})_{\mathcal{S}}=a \delta^{o}(\hat{A})_{\mathcal{S}}$. This is due the behaviour of spectral families under the mapping $\hat{A} \mapsto-\hat{A}$.

7. Let $\hat{A}$ be a self-adjoint operator, and let $\hat{E}[A \leq \lambda]=\hat{E}_{\lambda}^{A}$ be an element of the spectral family of $\hat{A}$. From (2.15) we get

$$
\hat{E}\left[\delta_{\mathcal{S}}^{o}(A) \leq \lambda\right]=\delta_{\mathcal{S}}^{i}(\hat{E}[A \leq \lambda])
$$


and then

$$
\begin{aligned}
\hat{E}\left[\delta^{o}(\hat{A})_{\mathcal{S}}>\lambda\right] & =\hat{1}-\hat{E}\left[\delta^{o}(\hat{A})_{\mathcal{S}} \leq \lambda\right] \\
& =\hat{1}-\delta_{\mathcal{S}}^{i}(\hat{E}[A \leq \lambda]) \\
& =\delta_{\mathcal{S}}^{o}(\hat{1}-\hat{E}[A \leq \lambda])
\end{aligned}
$$

where we have used the general result that, for any projection $\hat{P}$, we have $\hat{1}-\delta^{i}(\hat{P})_{\mathcal{S}}=$ $\delta_{\mathcal{S}}^{o}(\hat{1}-\hat{P})$. Then, (2.27) gives

$$
\hat{E}\left[\delta^{o}(\hat{A})_{\mathcal{S}}>\lambda\right]=\delta^{o}(\hat{E}[A>\lambda])_{\mathcal{S}} .
$$

\subsubsection{The de Groote Presheaves}

We know that $V \mapsto \delta^{o}(\hat{P})_{V}$ and $V \mapsto \delta^{i}(\hat{P})_{V}$ are global elements of the outer presheaf, $\underline{O}$, and inner presheaf, $\underline{I}$, respectively. Using the daseinisation operation for selfadjoint operators, it is straightforward to construct analogous presheaves for which $V \mapsto \delta^{o}(\hat{A})_{V}$ and $V \mapsto \delta^{i}(\hat{A})_{V}$ are global elements. One of these presheaves was briefly considered in [6]. We call these the 'de Groote presheaves' in recognition of the importance of de Groote's work.

Definition 2.5 The outer de Groote presheaf, $\underline{\mathbb{Q}}$, is defined as follows:

(i) On objects $V \in \operatorname{Ob}(\mathcal{V}(\mathcal{H})): \underline{\mathbb{Q}}_{V}:=V_{\mathrm{sa}}$, the collection of self-adjoint members of $V$.

(ii) On morphisms $i_{V^{\prime} V}: V^{\prime} \subseteq V$ : The mapping $\underline{\mathbb{Q}}\left(i_{V^{\prime} V}\right): \underline{\mathbb{Q}}_{V} \rightarrow \underline{\mathbb{Q}}_{V^{\prime}}$ is given by

$$
\begin{aligned}
\underline{\mathbb{Q}}\left(i_{V^{\prime} V}\right)(\hat{A}) & :=\delta^{o}(\hat{A})_{V^{\prime}} \\
& =\int_{\mathbb{R}} \lambda d\left(\delta^{i}\left(\hat{E}_{\lambda}^{A}\right)_{V^{\prime}}\right) \\
& =\int_{\mathbb{R}} \lambda d\left(\underline{I}\left(i_{V^{\prime} V}\right)\left(\hat{E}_{\lambda}^{A}\right)\right)
\end{aligned}
$$

for all $\hat{A} \in \underline{\mathbb{Q}}_{V}$.

Here we used the fact that the restriction mapping $\underline{I}\left(i_{V^{\prime} V}\right)$ of the inner presheaf $\underline{I}$ is the inner daseinisation of projections $\delta^{i}: \mathcal{P}(V) \rightarrow \mathcal{P}\left(V^{\prime}\right)$.

Definition 2.6 The inner de Groote presheaf, $\underline{\mathbb{I}}$, is defined as follows:

(i) On objects $V \in \operatorname{Ob}(\mathcal{V}(\mathcal{H})): \underline{\mathbb{I}}_{V}:=V_{\mathrm{sa}}$, the collection of self-adjoint members of $V$. 
(ii) On morphisms $i_{V^{\prime} V}: V^{\prime} \subseteq V:$ The mapping $\underline{\mathbb{I}}\left(i_{V^{\prime} V}\right): \underline{\mathbb{I}}_{V} \rightarrow \underline{\mathbb{I}}_{V^{\prime}}$ is given by

$$
\begin{aligned}
\underline{\mathbb{I}}\left(i_{V^{\prime} V}\right)(\hat{A}) & :=\delta^{i}(\hat{A})_{V^{\prime}} \\
& =\int_{\mathbb{R}} \lambda d\left(\bigwedge_{\mu>\lambda}\left(\delta^{o}\left(\hat{E}_{\mu}^{A}\right)_{V^{\prime}}\right)\right. \\
& =\int_{\mathbb{R}} \lambda d\left(\bigwedge_{\mu>\lambda}\left(\underline{O}\left(i_{V^{\prime} V}\right)\left(\hat{E}_{\mu}^{A}\right)\right)\right.
\end{aligned}
$$

for all $\hat{A} \in \underline{\mathbb{Q}}_{V}$ (where $\left.\underline{Q}\left(i_{V^{\prime} V}\right)=\delta^{o}: \mathcal{P}(V) \rightarrow \mathcal{P}\left(V^{\prime}\right)\right)$.

It is now clear that, by construction, $\delta^{o}(\hat{A}):=V \mapsto \delta^{o}(\hat{A})_{V}$ is a global element of $\underline{\mathbb{Q}}$, and $\delta^{i}(\hat{A}):=V \mapsto \delta^{i}(\hat{\hat{A}})_{V}$ is a global element of $\underline{\mathbb{I}}$.

De Groote found an example of an element of $\Gamma \underline{\underline{Q}}$ that is not of the form $\delta^{\circ}(\hat{A})$ (as mentioned in [6]). The same example can be used to show that there are global elements of the outer presheaf $\underline{O}$ that are not of the form $\delta^{o}(\hat{P})$ for any projection $\hat{P} \in \mathcal{P}(\mathcal{H})$.

\section{The Presheaves $\underline{\operatorname{sp}(\hat{A})^{\succeq}}$ and $\underline{\mathbb{R}^{\succeq}}$}

\subsection{Background to the Presheaf $\underline{\mathbb{R}^{\succeq}}$}

Our goal now is to construct a 'quantity-value' presheaf $\underline{\mathcal{R}}$ with the property that inner and/or outer daseinisation of an self-adjoint operator $\hat{A}$ can be used to define an arrow, i.e., a natural transformation, from $\underline{\Sigma}$ to $\underline{\mathcal{R}} 8$

The arrow corresponding to a self-adjoint operator $\hat{A} \in \mathcal{B}(\mathcal{H})$ is denoted for now by $\breve{A}: \underline{\Sigma} \rightarrow \underline{\mathcal{R}}$. At each stage $V$, we need a mapping

$$
\begin{aligned}
\breve{A}_{V}: \underline{\Sigma}_{V} & \rightarrow \underline{\mathcal{R}}_{V} \\
\lambda & \mapsto \breve{A}_{V}(\lambda)
\end{aligned}
$$

and we assume that this mapping is given by evaluation on a suitable member of $V$. More precisely, $\lambda \in \underline{\Sigma}_{V}$ is a spectral element $\ln ^{9}$ of $V$ and hence can be evaluated on operators lying in $V$. And, while $\hat{A}$ will generally not lie in $V$, both the inner daseinisation $\delta^{i}(\hat{A})_{V}$ and the outer daseinisation $\delta^{o}(\hat{A})_{V}$ do.

Let us start by considering the operators $\delta^{o}(\hat{A})_{V}, V \in \mathrm{Ob}(\mathcal{V}(\mathcal{H}))$. Each of these is a self-adjoint operator in the commutative von Neumann algebra $V$, and hence, by the spectral theorem, can be represented by a function, (the Gel'fand transform) $\overline{\delta^{o}(\hat{A})_{V}}: \underline{\Sigma}_{V} \rightarrow \operatorname{sp}\left(\delta^{o}(\hat{A})_{V}\right)$, with values in the spectrum $\operatorname{sp}\left(\delta^{o}(\hat{A})_{V}\right)$ of the self-adjoint

\footnotetext{
${ }^{8}$ In fact, we will define several closely related presheaves that can serve as a quantity-value object.

${ }^{9}$ In this context, by a 'spectral element', $\lambda \in \underline{\Sigma}_{V}$ of $V$, we mean a multiplicative, linear functional $\lambda: V \rightarrow \mathbb{C}$ with $\lambda(\hat{1})=1$.
} 
operator $\delta^{o}(\hat{A})_{V}$. Since the spectrum of a self-adjoint operator is a subset of $\mathbb{R}$, we can also write $\overline{\delta^{o}(\hat{A})_{V}}: \underline{\Sigma}_{V} \rightarrow \mathbb{R}$. The question now is whether the collection of maps $\overline{\delta^{o}(\hat{A})_{V}}: \underline{\Sigma}_{V} \rightarrow \mathbb{R}, V \in \mathrm{Ob}(\mathcal{V}(\mathcal{H}))$, can be regarded as an arrow from $\underline{\Sigma}$ to some presheaf $\underline{\mathcal{R}}$.

To answer this we need to see how these operators behave as we go 'down a chain' of subalgebras $V^{\prime} \subseteq V$. The first remark is that if $V^{\prime} \subseteq V$ then $\delta^{o}(\hat{A})_{V^{\prime}} \succeq \delta^{o}(\hat{A})_{V}$. When applied to the Gel'fand transforms, this leads to the equation

$$
\overline{\delta^{o}(\hat{A})_{V^{\prime}}}\left(\left.\lambda\right|_{V^{\prime}}\right) \geq \overline{\delta^{o}(\hat{A})_{V}}(\lambda)
$$

for all $\lambda \in \underline{\Sigma}_{V}$, where $\left.\lambda\right|_{V^{\prime}}$ denotes the restriction of the spectral element $\lambda \in \underline{\Sigma}_{V}$ to the subalgebra $V^{\prime} \subseteq V$. However, the definition of the spectral presheaf is such that $\left.\lambda\right|_{V^{\prime}}=\underline{\Sigma}\left(i_{V^{\prime} V}\right)(\lambda)$, and hence (3.3) can be rewritten as

$$
\overline{\delta^{o}(\hat{A})_{V^{\prime}}}\left(\underline{\Sigma}\left(i_{V^{\prime} V}\right)(\lambda)\right) \geq \overline{\delta^{o}(\hat{A})_{V}}(\lambda)
$$

for all $\lambda \in \underline{\Sigma}_{V}$.

It is a standard result that the real-number object, $\underline{\mathbb{R}}$, in a presheaf topos $\operatorname{Sets}^{\mathcal{C}^{o p}}$ is the constant functor from $\mathcal{C}$ to $\mathbb{R}$ [1]. It follows that the family of Gel'fand transforms, $\overline{\delta^{o}(\hat{A})_{V}}, V \in \mathrm{Ob}(\mathcal{V}(\mathcal{H}))$, of the daseinised operators $\delta^{o}(\hat{A})_{V}, V \in \mathrm{Ob}(\mathcal{V}(\mathcal{H}))$, cannot define an arrow from $\underline{\Sigma}$ to $\underline{\mathbb{R}}$, as this would require an equality in (3.4), which is not true. Thus the quantity-value presheaf, $\underline{\mathcal{R}}$, in the topos $\operatorname{Sets}^{\mathcal{V}(\mathcal{H})^{\mathrm{op}}}$ is not the realnumber object $\underline{\mathbb{R}}$, although clearly $\underline{\mathcal{R}}$ has something to do with the real numbers. We must take into account the growth of these real numbers as we go from $V$ to smaller subalgebras $V^{\prime}$. Similarly, if we consider inner daseinisation, we get a series of falling real numbers.

\subsection{Definition of the Presheaves $\underline{\operatorname{sp}(\hat{A})} \succeq$ and $\underline{\mathbb{R}} \succeq$}

The inapplicability of the real-number object $\underline{\mathbb{R}}$ may seem strange at first 10 but actually it is not that surprising. Because of the Kochen-Specker theorem, we do not expect to be able to assign (constant) real numbers as values of physical quantities, at least not globally. Instead, we draw on some recent results of M. Jackson [16], obtained as part of his extensive study of measure theory on a topos of presheaves. Here, we use a single construction in Jackson's thesis: the presheaf of 'order-preserving functions' over a partially ordered set - in our case, $\mathcal{V}(\mathcal{H})$. In fact, we will need both order-reversing and order-preserving functions.

Definition 3.1 Let $(\mathcal{Q}, \preceq)$ and $(\mathcal{P}, \preceq)$ be partially ordered sets. A function

$$
\mu: \mathcal{Q} \rightarrow \mathcal{P}
$$

\footnotetext{
${ }^{10}$ Indeed, it puzzled us for a while!
} 
is order-preserving if $q_{1} \preceq q_{2}$ implies $\mu\left(q_{1}\right) \preceq \mu\left(q_{2}\right)$ for all $q_{1}, q_{2} \in \mathcal{Q}$. It is orderreversing if $q_{1} \preceq q_{2}$ implies $\mu\left(q_{1}\right) \succeq \mu\left(q_{2}\right)$. We denote by $\mathcal{O P}(\mathcal{Q}, \mathcal{P})$ the set of orderpreserving functions $\mu: \mathcal{Q} \rightarrow \mathcal{P}$, and by $\mathcal{O R}(\mathcal{Q}, \mathcal{P})$ the set of order-reversing functions.

We note that if $\mu$ is order-preserving, then $-\mu$ is order-reversing, and vice versa.

Adapting Jackson's definitions slightly, if $\mathcal{P}$ is any partially-ordered set, we have the following.

Definition 3.2 The $\mathcal{P}$-valued presheaf, $\underline{\mathcal{P}}^{\succeq}$, of order-reversing functions over $\mathcal{V}(\mathcal{H})$ is defined as follows:

(i) On objects $V \in \mathrm{Ob}(\mathcal{V}(\mathcal{H}))$ :

$$
\underline{\mathcal{P}}_{V}^{\succ}:=\{\mu: \downarrow V \rightarrow \mathcal{P} \mid \mu \in \mathcal{O} \mathcal{R}(\downarrow V, \mathcal{P}\}
$$

where $\downarrow V \subset \operatorname{Ob}(\mathcal{V}(\mathcal{H}))$ is the set of all unital von Neumann subalgebras of $V$.

(ii) On morphisms $i_{V^{\prime} V}: V^{\prime} \subseteq V$ : The mapping $\underline{\mathcal{P}}^{\succeq}\left(i_{V^{\prime} V}\right): \underline{\mathcal{P}}_{V}^{\succeq} \rightarrow \underline{\mathcal{P}}_{V^{\prime}}^{\succeq}$ is given by

$$
\underline{\mathcal{P}}^{\succeq}\left(i_{V^{\prime} V}\right)(\mu):=\mu_{V_{V^{\prime}}}
$$

where $\mu_{V_{V^{\prime}}}$ denotes the restriction of the function $\mu$ to $\downarrow V^{\prime} \subseteq \downarrow V$.

Jackson uses order-preserving functions with $\mathcal{P}:=[0, \infty)$ (the non-negative reals), with the usual order $\leq$.

Clearly, there is an analogous definition of the $\mathcal{P}$-valued presheaf, $\underline{\mathcal{P}}^{\leq}$, of orderpreserving functions from $\downarrow V$ to $\mathcal{P}$. It can be shown that $\underline{\mathcal{P}}^{\succeq}$ and $\underline{\mathcal{P}}^{\preceq}$ are isomorphic

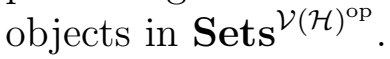

Let us first consider $\underline{\mathcal{P}}^{\succeq}$. For us, the key examples for the partially ordered set $\mathcal{P}$ are (i) $\mathbb{R}$, the real numbers with the usual order $\leq$, and (ii) $\operatorname{sp}(\hat{\mathrm{A}}) \subset \mathbb{R}$, the spectrum of some bounded self-adjoint operator $\hat{A}$, with the order $\leq$ inherited from $\mathbb{R}$. Clearly, the associated presheaf $\operatorname{sp}(\hat{A}) \succeq$ is a sub-object of the presheaf $\underline{\mathbb{R}^{\succeq}}$.

Now let $\hat{A} \in \mathcal{B}(\mathcal{H})_{\text {sa }}$, and let $V \in \operatorname{Ob}(\mathcal{V}(\mathcal{H}))$. Then to each $\lambda \in \underline{\Sigma}_{V}$ there is associated the function

$$
\breve{\delta}^{o}(A)_{V}(\lambda): \downarrow V \rightarrow \operatorname{sp}(\hat{A}),
$$

given by

$$
\begin{aligned}
\left(\breve{\delta}^{o}(A)_{V}(\lambda)\right)\left(V^{\prime}\right) & :=\overline{\delta^{o}(\hat{A})_{V^{\prime}}}\left(\underline{\Sigma}\left(i_{V^{\prime} V}\right)(\lambda)\right) \\
& =\overline{\delta^{o}(\hat{A})_{V^{\prime}}}\left(\left.\lambda\right|_{V^{\prime}}\right) \\
& =\left.\lambda\right|_{V^{\prime}}\left(\delta^{o}(\hat{A})_{V^{\prime}}\right) \\
& =\lambda\left(\delta^{o}(\hat{A})_{V^{\prime}}\right)
\end{aligned}
$$


for all $V^{\prime} \subseteq V$. We note that as $V^{\prime}$ becomes smaller, $\delta^{o}(\hat{A})_{V^{\prime}}$ becomes larger (or stays the same) in the spectral order, and hence in the usual order on operators. Therefore, $\breve{\delta}^{o}(A)_{V}(\lambda): \downarrow V \rightarrow \operatorname{sp}(\hat{A})$ is an order-reversing function, for each $\lambda \in \underline{\Sigma}_{V}$.

Let

$$
\begin{aligned}
\breve{\delta}^{o}(A)_{V}: \underline{\Sigma}_{V} & \rightarrow \frac{\operatorname{sp}(\hat{A})^{\succeq}}{\lambda} \\
\lambda & \mapsto \breve{\delta}^{o}(A)_{V}(\lambda)
\end{aligned}
$$

denote the set of order-reversing functions from $\downarrow V$ to $\operatorname{sp}(\hat{A})$ obtained in this way.

Theorem 3.1 The mappings $\breve{\delta}^{o}(A)_{V}, V \in \mathrm{Ob}(\mathcal{V}(\mathcal{H}))$, are the components of a natural transformation/arrow $\breve{\delta}^{o}(A): \underline{\Sigma} \rightarrow \underline{\operatorname{sp}(\hat{A}) \succeq}$.

Proof. We only have to prove that, whenever $V^{\prime} \subset V$, the diagram

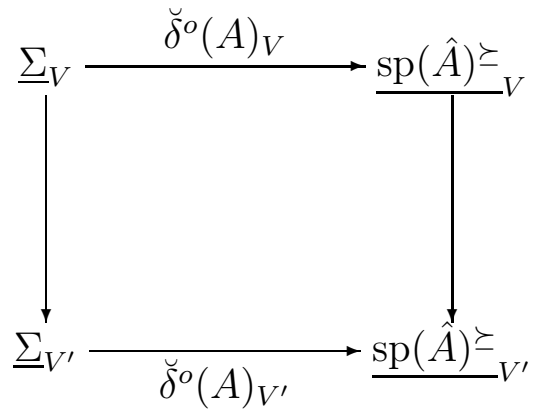

commutes. Here, the vertical arrows are the restrictions of the relevant presheaves from the stage $V$ to $V^{\prime} \subset V$.

In fact, the commutativity of the diagram follows directly from the definitions. For each $\lambda \in \underline{\Sigma}_{V}$, the composition of the upper arrow and the right vertical arrow gives

$$
\left.\left(\breve{\delta}^{o}(A)_{V}(\lambda)\right)\right|_{V^{\prime}}=\breve{\delta}^{o}(A)_{V^{\prime}}\left(\left.\lambda\right|_{V^{\prime}}\right)
$$

which is the same function that we get by first restricting $\lambda$ from $\underline{\Sigma}_{V}$ to $\underline{\Sigma}_{V^{\prime}}$ and then applying $\breve{\delta}^{o}(A)_{V^{\prime}}$.

In this way, to each physical quantity $\hat{A}$ in quantum theory there is assigned a natural transformation $\breve{\delta}^{o}(A)$ from the state object $\underline{\Sigma}$ to the presheaf $\operatorname{sp}(\hat{A})^{\succeq}$. Since $\operatorname{sp}(\hat{A})^{\succeq}$ is a sub-object of $\underline{\mathbb{R}^{\succeq}}$ for each $\hat{A}, \breve{\delta}^{o}(A)$ can also be seen as a natural transfor-

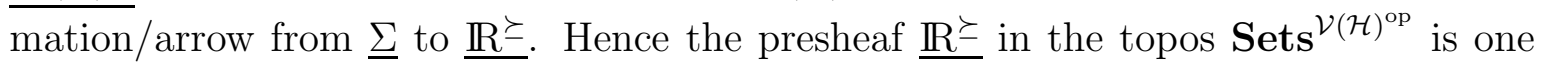
candidate for the quantity-value object of quantum theory.

In the Appendix, we show that the mapping

$$
\begin{aligned}
\theta: \mathcal{B}(\mathcal{H})_{\mathrm{sa}} & \rightarrow \operatorname{Hom}_{\operatorname{Sets} \mathcal{V}(\mathcal{H})^{\mathrm{op}}}(\underline{\Sigma}, \underline{\mathbb{R}}) \\
\hat{A} & \mapsto \breve{\delta}^{o}(A)
\end{aligned}
$$


is injective. Furthermore, if $S$ denotes our quantum system, then, on the level of the language $\mathcal{L}(S)$, we expect the mapping $A \rightarrow \hat{A}$ to be injective, where $A$ is a function symbol of signature $\Sigma \rightarrow \mathcal{R}$. It follows that we have obtained a faithful representation of these function symbols by arrows $\breve{\delta}^{o}(A): \underline{\Sigma} \rightarrow \underline{\mathbb{R}^{\succeq}}$ in the topos $\operatorname{Sets}{ }^{\mathcal{V}(\mathcal{H})^{\text {op }}}$.

Similarly, there is an order-preserving function

$$
\breve{\delta}^{i}(A)_{V}(\lambda): \downarrow V \rightarrow \operatorname{sp}(\hat{A}),
$$

that is defined for all $V^{\prime} \subseteq V$ by

$$
\begin{aligned}
\left(\breve{\delta}^{i}(A)_{V}(\lambda)\right)\left(V^{\prime}\right) & =\overline{\delta^{i}(\hat{A})_{V^{\prime}}}\left(\underline{\Sigma}\left(i_{V^{\prime} V}\right)(\lambda)\right) \\
& =\lambda\left(\delta^{i}(\hat{A})_{V^{\prime}}\right) .
\end{aligned}
$$

Since $\delta^{i}(\hat{A})_{V^{\prime}}$ becomes smaller (or stays the same) as $V^{\prime}$ gets smaller, $\breve{\delta}^{i}(A)_{V}(\lambda)$ indeed is an order-preserving function from $\downarrow V$ to $\operatorname{sp}(\hat{A})$ for each $\lambda \in \underline{\Sigma}_{V}$.

Clearly, we can use these functions to define a natural transformation $\breve{\delta}^{i}(A): \underline{\Sigma} \rightarrow$ $\underline{\mathbb{R}} \preceq$ from the spectral presheaf, $\underline{\Sigma}$, to the presheaf $\underline{\mathbb{R}} \preceq$ of real-valued, order-preserving functions on $\downarrow V$. The components of $\breve{\delta}^{i}(A)$ are

$$
\begin{aligned}
\breve{\delta}^{i}(A)_{V}: \underline{\Sigma}_{V} & \rightarrow \frac{\mathrm{sp}(\hat{\mathrm{A}})^{\preceq}}{\breve{\delta}^{i}(A)_{V}(\lambda) .} \\
\lambda & \mapsto \sin
\end{aligned}
$$

The functions obtained from inner and outer daseinisation can be combined to give yet another presheaf, and one that will be particularly useful for the physical interpretation of these constructions. The general definition is:

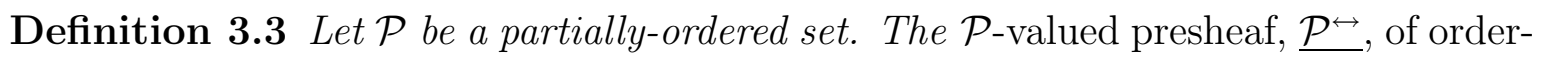
preserving and order-reversing functions on $\mathcal{V}(\mathcal{H})$ is defined as follows:

(i) On objects $V \in O b(\mathcal{V}(\mathcal{H}))$ :

$$
\underline{\mathcal{P}}_{V}^{\leftrightarrow}:=\{(\mu, \nu) \mid \mu \in \mathcal{O P}(\downarrow V, \mathcal{P}), \nu \in \mathcal{O} \mathcal{R}(\downarrow V, \mathcal{P})\},
$$

where $\downarrow V \subset \mathrm{Ob}(\mathcal{V}(\mathcal{H}))$ is the set of all subalgebras $V^{\prime}$ of $V$.

(ii) On morphisms $i_{V^{\prime} V}: V^{\prime} \subseteq V$ :

$$
\begin{aligned}
\underline{\mathcal{P}^{\leftrightarrow}}\left(i_{V^{\prime} V}\right): \frac{\mathcal{P}^{\leftrightarrow}}{V} & \longrightarrow \frac{\mathcal{P}^{\leftrightarrow}}{V^{\prime}} \\
(\mu, \nu) & \longmapsto\left(\left.\mu\right|_{V^{\prime}},\left.\nu\right|_{V^{\prime}}\right),
\end{aligned}
$$

where $\left.\mu\right|_{V^{\prime}}$ denotes the restriction of $\mu$ to $\downarrow V^{\prime} \subseteq \downarrow V$, and analogously for $\left.\nu\right|_{V^{\prime}}$.

As we will see shortly, the presheaf, $\underline{\mathbb{R}} \leftrightarrow$, of order-preserving and order-reversing, real-valued functions is closely related to the ' $k$-extension' of the presheaf $\underline{\mathbb{R}^{\succeq}}$.

Now let

$$
\breve{\delta}(A)_{V}:=\left(\breve{\delta}^{i}(A)_{V}(\cdot), \breve{\delta}^{o}(A)_{V}(\cdot)\right): \underline{\Sigma}_{V} \rightarrow \underline{\mathbb{R}}^{\leftrightarrow} V
$$


denote the set of all pairs of order-preserving and order-reversing functions from $\downarrow V$ to $\mathbb{R}$ that can be obtained from inner and outer daseinisation. It is easy to see that we have the following result:

Theorem 3.2 The mappings $\breve{\delta}(A)_{V}, V \in \mathrm{Ob}(\mathcal{V}(\mathcal{H}))$, are the components of a natural transformation $\breve{\delta}(A): \underline{\Sigma} \rightarrow \underline{\mathbb{R} \leftrightarrow}$.

\subsection{Inner and Outer Daseinisation from Functions on Filters}

There is a close relationship between inner and outer daseinisation, and certain functions on the dual ideals/filters in the projection lattice $\mathcal{P}(\mathcal{H})$ of $\mathcal{B}(\mathcal{H})$. We only give a very brief sketch here: details can be found in de Groote's work [6, 7], the article [9], and a forthcoming paper [10]. This subsection serves as a preparation for the physical interpretation of the arrows $\breve{\delta}(A): \underline{\Sigma} \rightarrow \underline{\mathbb{R}^{\leftrightarrow}}$.

Spectral elements and ultrafilters. Let $V \in \mathrm{Ob}(\mathcal{V}(\mathcal{H}))$, and let $\lambda \in \underline{\Sigma}_{V}$ be a spectral element of the von Neumann algebra $V$. For all projections $\hat{P} \in \mathcal{P}(V)$, we have

$$
\lambda(\hat{P})=\lambda\left(\hat{P}^{2}\right)=\lambda(\hat{P}) \lambda(\hat{P}),
$$

and so $\lambda(\hat{P}) \in\{0,1\}$. Moreover, $\lambda(\hat{0})=0, \lambda(\hat{1})=1$, and if $\lambda(\hat{P})=0$, then $\lambda(\hat{1}-\hat{P})=1$ (since $\lambda(\hat{P})+\lambda(\hat{1}-\hat{P})=\lambda(\hat{1}))$. Hence, for each $\hat{P} \in \mathcal{P}(V)$ we have either $\lambda(\hat{P})=1$ or $\lambda(\hat{1}-\hat{P})=1$. The family

$$
F_{\lambda}:=\{\hat{P} \in \mathcal{P}(V) \mid \lambda(\hat{P})=1\}
$$

is an ultrafilter in $\mathcal{P}(V) 11$ Conversely, each $\lambda \in \underline{\Sigma}_{V}$ is uniquely determined by the set $\{\lambda(\hat{P}) \mid \hat{P} \in \mathcal{P}(V)\}$ and hence by an ultrafilter in $\mathcal{P}(V)$. This shows that there is a bijection between the set $\mathcal{Q}(V)$ of ultrafilters in $\mathcal{P}(V)$ and the Gel'fand spectrum $\underline{\Sigma}_{V}$.

Given a filter base $F$ in $\mathcal{P}(\mathcal{H})$, the cone over $F$ in $\mathcal{B}(\mathcal{H})$ is defined as

$$
\mathcal{C}_{\mathcal{B}(\mathcal{H})}(F):=\{\hat{Q} \in \mathcal{P}(\mathcal{H}) \mid \exists \hat{P} \in F: \hat{P} \preceq \hat{Q}\}
$$

This is the smallest filter in $\mathcal{P}(\mathcal{H})$ that contains the filter base $F$. We write $\mathcal{C}(F)$ for $\mathcal{C}_{\mathcal{B}(\mathcal{H})}(F)$.

Observable and antonymous functions. Let $\mathcal{N}$ be a unital von Neumann algebra, and let $\mathcal{D}(\mathcal{N})$ be the set of filters in the projection lattice $\mathcal{P}(\mathcal{N})$ of $\mathcal{N}$. De Groote has shown [7] that to each self-adjoint operator $\hat{A} \in \mathcal{N}$, there corresponds a, so-called,

\footnotetext{
${ }^{11}$ Let $\mathbb{L}$ be a lattice with zero element 0 . A subset $F \subset \mathbb{L}$ is a 'filter base' if (i) $0 \notin F$ and (ii) for all $a, b \in F$, there is some $c \in F$ such that $c \leq a \wedge b$. A subset $D \subset \mathbb{L}$ is called a '(proper) dual ideal' or a 'filter' if (i) $0 \notin D$, (ii) for all $a, b \in D, a \wedge b \in D$ and (iii) $a \in D$ and $b>a$ implies $b \in D$. A maximal dual ideal/filter $F$ in a complemented, distributive lattice $\mathbb{L}$ is called an 'ultrafilter'. It has the property that for all $a \in \mathbb{L}$, either $a \in F$ or $a^{\prime} \in F$, where $a^{\prime}$ is the complement of $a$.
} 
'observable function' $f_{\hat{A}}: \mathcal{D}(\mathcal{N}) \rightarrow \operatorname{sp}(\hat{A})$. If $\mathcal{N}$ is abelian, $\mathcal{N}=V$, then $\left.f_{\hat{A}}\right|_{\mathcal{Q}(V)}$ is just the Gel'fand transform of $\hat{A}$. However, it is striking that $f_{\hat{A}}$ can be defined even if $\mathcal{N}$ is non-abelian; for us, the important example is $\mathcal{N}=\mathcal{B}(\mathcal{H})$.

If $\left\{\hat{E}_{\mu}^{A}\right\}_{\mu \in \mathbb{R}}$ is the spectral family of $\hat{A}$, then $f_{\hat{A}}$ is defined as

$$
\begin{aligned}
f_{\hat{A}}: \mathcal{D}(\mathcal{N}) & \rightarrow \operatorname{sp}(\hat{A}) \\
D & \mapsto \inf \left\{\mu \in \mathbb{R} \mid \hat{E}_{\mu}^{A} \in D\right\} .
\end{aligned}
$$

Conversely, given a bounded function $f: \mathcal{D}(\mathcal{H}) \rightarrow \mathbb{R}$ with certain properties, one can find a unique self-adjoint operator $\hat{A} \in \mathcal{B}(\mathcal{H})$ such that $f=f_{\hat{A}}$.

Prop. 3.1 in [6] shows that, for all $V \in \operatorname{Ob}(\mathcal{V}(\mathcal{H}))$ and all filters $D$ in $\mathcal{D}(V)$,

$$
f_{\delta^{o}(\hat{A})_{V}}(D)=f_{\hat{A}}(\mathcal{C}(\mathcal{I})) \text {. }
$$

We saw above that to each $\lambda \in \underline{\Sigma}_{V}$ there corresponds a unique ultrafilter $F_{\lambda} \in \mathcal{Q}(V) 12$ Since $\delta^{o}(\hat{A})_{V} \in V$, the observable function $f_{\delta^{o}(\hat{A})_{V}}$ is the Gel'fand transform of $\delta^{o}(\hat{A})_{V}$, and so, on identifying the ultrafilter $F_{\lambda}$ with the spectral element $\lambda$, we have

$$
f_{\delta^{o}(\hat{A})_{V}}\left(F_{\lambda}\right)=\overline{\delta^{o}(\hat{A})_{V}}(\lambda)=\lambda\left(\delta^{o}(\hat{A})_{V}\right) .
$$

From (3.31) we have

$$
\lambda\left(\delta^{o}(\hat{A})_{V}\right)=f_{\delta^{o}(\hat{A})_{V}}\left(F_{\lambda}\right)=f_{\hat{A}}\left(\mathcal{C}\left(F_{\lambda}\right)\right)
$$

for all $V \in \operatorname{Ob}(\mathcal{V}(\mathcal{H}))$ and for all $\lambda \in \underline{\Sigma}_{V}$. In this sense, the observable function $f_{\hat{A}}$ encodes all the outer daseinisations $\delta^{o}(\hat{A})_{V}, V \in \mathrm{Ob}(\mathcal{V}(\mathcal{H}))$, of $\hat{A}$.

There is also a function, $g_{\hat{A}}$, on the filters in $\mathcal{P}(\mathcal{H})$ that encodes all the inner daseinisations $\delta^{i}(\hat{A})_{V}, V \in \mathrm{Ob}(\mathcal{V}(\mathcal{H}))$. This function is given for an arbitrary unital von Neumann algebra $\mathcal{N}$ by

$$
\begin{aligned}
g_{\hat{A}}: \mathcal{D}(\mathcal{N}) & \rightarrow \operatorname{sp}(\hat{A}) \\
D & \mapsto \sup \left\{\lambda \in \mathbb{R} \mid \hat{1}-\hat{E}_{\lambda}^{A} \in D\right\}
\end{aligned}
$$

and is called the 'antonymous function' of $\hat{A}[9]$. If $\mathcal{N}$ is abelian, then $g_{\hat{A}}$ is the Gel'fand transform of $\hat{A}$ (and coincides with $f_{\hat{A}}$ ). One can show [10] that for all $V \in \mathrm{Ob}(\mathcal{V}(\mathcal{H})$ ) and all filters $D$ in $\mathcal{D}(V)$,

$$
g_{\delta^{i}(\hat{A})_{V}}(D)=g_{\hat{A}}(\mathcal{C}(\mathcal{I}))
$$

Let $\lambda \in \underline{\Sigma}_{V}$, and let $F_{\lambda} \in \mathcal{Q}(V)$ be the corresponding ultrafilter. Since $\delta^{i}(\hat{A})_{V} \in V$, the antonymous function $g_{\delta^{i}(\hat{A})_{V}}$ is the Gel'fand transform of $\delta^{i}(\hat{A})_{V}$, and we have

$$
g_{\delta^{i}(\hat{A})_{V}}\left(F_{\lambda}\right)=\overline{\delta^{i}(\hat{A})_{V}}(\lambda)=\lambda\left(\delta^{i}(\hat{A})_{V}\right)
$$

From (3.36), we get

$$
\lambda\left(\delta^{i}(\hat{A})_{V}\right)=g_{\delta^{i}(\hat{A})_{V}}\left(F_{\lambda}\right)=g_{\hat{A}}\left(\mathcal{C}\left(F_{\lambda}\right)\right)
$$

for all $V \in \operatorname{Ob}(\mathcal{V}(\mathcal{H}))$ and all $\lambda \in \underline{\Sigma}_{V}$. Thus the antonymous function $g_{\hat{A}}$ encodes all the inner daseinisations $\delta^{i}(\hat{A})_{V}, V \in \mathrm{Ob}(\mathcal{V}(\mathcal{H}))$, of $\hat{A}$.

\footnotetext{
${ }^{12}$ De Groote calls the maximal dual ideals (i.e., maximal filters) in an arbitrary lattice the 'quasipoints' of the lattice.
} 


\subsection{A Physical Interpretation of the Arrow $\breve{\delta}(A): \underline{\Sigma} \rightarrow \underline{\mathbb{R}^{\leftrightarrow}}$}

Let $|\psi\rangle \in \mathcal{H}$ be a unit vector in the Hilbert space of the quantum system. The expectation value of a self-adjoint operator $\hat{A} \in \mathcal{B}(\mathcal{H})$ in the state $|\psi\rangle$ is given by

$$
\langle\psi|\hat{A}| \psi\rangle=\int_{-\|\hat{A}\|}^{\|\hat{A}\|} \lambda d\left\langle\psi\left|\hat{E}_{\lambda}^{A}\right| \psi\right\rangle .
$$

There is a maximal filter $F_{|\psi\rangle}$ in $\mathcal{P}(\mathcal{H}), 13$ given by

$$
F_{|\psi\rangle}:=\left\{\hat{P} \in \mathcal{P}(\mathcal{H}) \mid \hat{P} \succeq \hat{P}_{|\psi\rangle}\right\},
$$

where $\hat{P}_{|\psi\rangle}$ is the projection onto the one-dimensional subspace of $\mathcal{H}$ generated by $|\psi\rangle$. As shown in [9], the expectation value $\langle\psi|\hat{A}| \psi\rangle$ can be written as

$$
\langle\psi|\hat{A}| \psi\rangle=\int_{g_{\hat{A}}\left(F_{|\psi\rangle}\right)}^{f_{\hat{A}}\left(F_{|\psi\rangle}\right)} \lambda d\left\langle\psi\left|\hat{E}_{\lambda}^{A}\right| \psi\right\rangle .
$$

In an instrumentalist interpretation 14 one would interpret $g_{\hat{A}}\left(F_{|\psi\rangle}\right)$, resp. $f_{\hat{A}}\left(F_{|\psi\rangle}\right)$, as the smallest, resp. largest, possible result of a measurement of the physical quantity $A$ when the state is $|\psi\rangle$. If $|\psi\rangle$ is an eigenstate of $\hat{A}$, then $\langle\psi|\hat{A}| \psi\rangle$ is an eigenvalue of $\hat{A}$, and in this case, $\langle\psi|\hat{A}| \psi\rangle \in \operatorname{sp}(\hat{A})$; moreover,

$$
\langle\psi|\hat{A}| \psi\rangle=g_{\hat{A}}\left(F_{|\psi\rangle}\right)=f_{\hat{A}}\left(F_{|\psi\rangle}\right) .
$$

If $|\psi\rangle$ is not an eigenstate of $\hat{A}$, then

$$
g_{\hat{A}}\left(F_{|\psi\rangle}\right)<\langle\psi|\hat{A}| \psi\rangle<f_{\hat{A}}\left(F_{|\psi\rangle}\right) .
$$

Let $V$ be an abelian subalgebra of $\mathcal{B}(\mathcal{H})$ such that $\underline{\Sigma}_{V}$ contains the spectral element, $\lambda^{|\psi\rangle}$, associated with $|\psi\rangle, 15$ The corresponding ultrafilter $\tilde{F}_{|\psi\rangle}$ in $\mathcal{P}(V)$ consists of those projections $\hat{Q} \in \mathcal{P}(V)$ such that $\hat{Q} \succeq \hat{P}_{|\psi\rangle}$. Hence the cone $\mathcal{C}\left(\tilde{F}_{|\psi\rangle}\right)$ consists of all projections $\hat{R} \in \mathcal{P}(\mathcal{H})$ such that $\hat{R} \succeq \hat{P}_{|\psi\rangle}$; and so

$$
\mathcal{C}\left(\tilde{F}_{|\psi\rangle}\right)=F_{|\psi\rangle} \text {. }
$$

This allows us to write the expectation value as

$$
\begin{aligned}
\langle\psi|\hat{A}| \psi\rangle & =\int_{g_{\hat{A}}\left(\mathcal{C}\left(\tilde{F}_{|\psi\rangle}\right)\right)}^{f_{\hat{A}}\left(\mathcal{C}\left(\tilde{F}_{|\psi\rangle}\right)\right)} \lambda d\left\langle\psi\left|\hat{E}_{\lambda}^{A}\right| \psi\right\rangle \\
& =\int_{g_{\delta^{i}(\hat{A})_{V}}\left(\tilde{F}_{|\psi\rangle}\right)}^{f_{\delta o}(\hat{A})_{V}\left(\tilde{F}_{|\psi\rangle}\right)} \lambda d\left\langle\psi\left|\hat{E}_{\lambda}^{A}\right| \psi\right\rangle .
\end{aligned}
$$

\footnotetext{
${ }^{13}$ Since $\mathcal{P}(\mathcal{H})$ is not distributive, $F_{|\psi\rangle}$ is not an ultrafilter; i.e., there are projections $\hat{P} \in \mathcal{P}(\mathcal{H})$ such that neither $\hat{P} \in F_{|\psi\rangle}$ nor $\hat{1}-\hat{P} \in F_{|\psi\rangle}$.

${ }^{14}$ Which we avoid in general, of course!

${ }^{15}$ This is the element defined by $\lambda^{|\psi\rangle}(\hat{A}):=\langle\psi|\hat{A}| \psi\rangle$ for all $\hat{A} \in V$.
} 
Equations (3.32) and (3.37) show that $f_{\delta^{o}(\hat{A})_{V}}\left(\tilde{F}_{|\psi\rangle}\right)=\left\langle\psi\left|\delta^{o}(\hat{A})_{V}\right| \psi\right\rangle$ and $g_{\delta^{i}(\hat{A})_{V}}\left(\tilde{F}_{|\psi\rangle}\right)=\left\langle\psi\left|\delta^{i}(\hat{A})_{V}\right| \psi\right\rangle$. In the language of instrumentalism, for stages $V$ for which $\lambda^{|\psi\rangle} \in \underline{\Sigma}_{V}$, the value $\left\langle\psi\left|\delta^{i}(\hat{A})_{V}\right| \psi\right\rangle \in \operatorname{sp}(\hat{A})$ is the smallest possible measurement result for $\hat{A}$ in the quantum state $|\psi\rangle$; and $\left\langle\psi\left|\delta^{o}(\hat{A})_{V}\right| \psi\right\rangle \in \operatorname{sp}(\hat{A})$ is the largest possible result.

If $\lambda \in \underline{\Sigma}_{V}$ is not of the form $\lambda=\lambda^{|\psi\rangle}$, then the cone $\mathcal{C}\left(F_{\lambda}\right)$ over the ultrafilter $F_{\lambda}$ corresponding to $\lambda$ cannot be identified with a vector in $\mathcal{H}$. Nevertheless, the quantity $\mathcal{C}\left(F_{\lambda}\right)$ is well-defined, and (3.31) and (3.36) hold. If we go from $V$ to a subalgebra $V^{\prime} \subset V$, then $\delta^{i}(\hat{A})_{V^{\prime}} \preceq \delta^{i}(\hat{A})_{V}$ and $\delta^{o}(\hat{A})_{V^{\prime}} \succeq \delta^{o}(\hat{A})_{V}$, hence

$$
\begin{aligned}
& \lambda\left(\delta^{i}(\hat{A})_{V^{\prime}}\right) \leq \lambda\left(\delta^{i}(\hat{A})_{V}\right), \\
& \lambda\left(\delta^{o}(\hat{A})_{V^{\prime}}\right) \geq \lambda\left(\delta^{o}(\hat{A})_{V}\right)
\end{aligned}
$$

for all $\lambda \in \underline{\Sigma}_{V}$.

We can interpret the function

$$
\begin{aligned}
\breve{\delta}(A)_{V}: \underline{\Sigma}_{V} & \rightarrow \underline{\mathbb{R}}_{V} \\
\lambda & \mapsto \breve{\delta}(A)_{V}(\lambda)=\left(\breve{\delta}^{i}(A)_{V}(\lambda), \breve{\delta}^{o}(A)_{V}(\lambda)\right)
\end{aligned}
$$

as giving the 'spread' or 'range' of the physical quantity $A$ at stages $V^{\prime} \subseteq V$. Each element $\lambda \in \underline{\Sigma}_{V}$ gives its own 'spread' $\breve{\delta}(A)_{V}(\lambda): \downarrow V \rightarrow \operatorname{sp}(\hat{A}) \times \operatorname{sp}(\hat{A})$. The intuitive idea is that at stage $V$, given a point $\lambda \in \underline{\Sigma}_{V}$, the physical quantity $A$ 'spreads over' the subset

$$
\left.\left[\breve{\delta}^{i}(A)_{V}(\lambda)(V), \breve{\delta}^{o}(A)_{V}(\lambda)(V)\right] \cap \operatorname{sp}(\hat{A})=\left[\lambda\left(\delta^{i}(\hat{A})_{V}\right), \lambda\left(\delta^{o}(\hat{A})\right)_{V}\right)\right] \cap \operatorname{sp}(\hat{A}),
$$

and for $V^{\prime} \subset V$, over the (potentially larger) subset

$$
\left[\breve{\delta}^{i}(A)_{V}(\lambda)\left(V^{\prime}\right), \breve{\delta}^{o}(A)_{V}(\lambda)\left(V^{\prime}\right)\right] \cap \operatorname{sp}(\hat{A})=\left[\lambda\left(\delta^{i}(\hat{A})_{V^{\prime}}, \lambda\left(\delta^{o}(\hat{A})\right)_{V^{\prime}}\right] \cap \operatorname{sp}(\hat{A}) .\right.
$$

All this is local in the sense that these expressions are defined at a stage $V$ and for subalgebras, $V^{\prime}$, of $V$, where $\lambda \in \underline{\Sigma}_{V}$. No similar global construction or interpretation is possible, since the spectral presheaf $\underline{\Sigma}$ has no global elements, i.e., no points (while the set $\underline{\Sigma}_{V}$ does have points).

As we go down to smaller subalgebras $V^{\prime} \subseteq V$, the spread gets larger. This comes from the fact that $\hat{A}$ has to be adopted more and more as we go to smaller subalgebras $V^{\prime}$. More precisely, $\hat{A}$ is approximated from below by $\delta^{i}(\hat{A})_{V^{\prime}} \in V^{\prime}$ and from above by $\delta^{o}(\hat{A})_{V^{\prime}} \in V^{\prime}$. This approximation gets coarser as $V^{\prime}$ gets smaller, which basically means that $V^{\prime}$ contains less and less projections.

It should be remarked that $\breve{\delta}(A)$ does not assign actual values to the physical quantity $A$, but rather the possible range of such values; and these are independent of any state $|\psi\rangle$. This is analogous to the classical case where physical quantities are represented by real-valued functions on state space. The range of possible values is state-independent, but the actual value possessed by a physical quantity does depend on the state of the system. 


\subsection{Properties of $\underline{\mathbb{R}^{\succeq}}$.}

From the perspective of our overall programme, Theorem 3.1 is a key result and suggests strongly that $\underline{\mathbb{R}} \succeq$ is an appropriate choice for the quantity-value object for quantum theory. To explore this further, we note the following elementary properties of $\underline{\mathbb{R}} \geq 16$

1. The presheaf $\underline{\mathbb{R}^{\succeq}}$ has global elements: namely, order-reversing functions on the partially-ordered set $\operatorname{Ob}(\mathcal{V}(\mathcal{H}))$ of objects in the category $\mathcal{V}(\mathcal{H})$; i.e., functions $\mu: \operatorname{Ob}(\mathcal{V}(\mathcal{H})) \rightarrow \mathbb{R}$ such that:

For all $V_{1}, V_{2} \in \mathrm{Ob}(\mathcal{V}(\mathcal{H})), V_{2} \subseteq V_{1}$ implies $\mu\left(V_{2}\right) \geq \mu\left(V_{1}\right)$.

2. (a) Elements of $\Gamma \underline{\mathbb{R}^{\succeq}}$ can be added: i.e., if $\mu, \nu \in \Gamma \underline{\mathbb{R}^{\succeq}}$, define $\mu+\nu$ at each stage $V$ by

$$
(\mu+\nu)\left(V^{\prime}\right):=\mu\left(V^{\prime}\right)+\nu\left(V^{\prime}\right)
$$

for all $V^{\prime} \subseteq V$. Note that if $V_{2} \subseteq V_{1} \subseteq V$, then $\mu\left(V_{1}\right) \leq \mu\left(V_{2}\right)$ and $\nu\left(V_{1}\right) \leq \nu\left(V_{2}\right)$, and so $\mu\left(V_{1}\right)+\nu\left(V_{1}\right) \leq \mu\left(V_{2}\right)+\nu\left(V_{2}\right)$. Thus the definition of $\mu+\nu$ in (3.54) makes sense. Obviously, addition is commutative and associative.

(b) However, it is not possible to define ' $\mu-\nu$ ' in this way since the difference between two order-reversing functions may not be order-reversing. This problem is addressed in Section 4 .

(c) A 'zero/unit' element can be defined for the additive structure on $\Gamma \underline{\mathbb{R}^{\succeq}}$ as $0(V):=0$ for all $V \in \operatorname{Ob}(\mathcal{V}(\mathcal{H}))$, where 0 denotes the function that is constantly 0 on $\mathrm{Ob}(\mathcal{V}(\mathcal{H}))$.

It follows from (a) and (c) that $\Gamma \underline{\mathbb{R}^{\succeq}}$ is a commutative monoid (i.e., a semigroup with a unit).

The commutative monoid structure for $\Gamma \underline{\mathbb{R}^{\succeq}}$ is a reflection of the stronger fact that $\underline{\mathbb{R}^{\succeq}}$ is a commutative-monoid object in the topos $\operatorname{Sets}^{\mathcal{V}(\mathcal{H})^{\mathrm{op}}}$. Specifically, there is an arrow $+: \underline{\mathbb{R}^{\succeq}} \times \underline{\mathbb{R}^{\succeq}} \rightarrow \underline{\mathbb{R}^{\succeq}}$ defined by ${ }_{V}(\mu, \nu):=\mu+\nu$ for all $\mu, \nu \in \underline{\mathbb{R}}_{V}$, and for all stages $V \in \overline{\mathrm{Ob}}(\mathcal{V}(\mathcal{H}))$. Here, $\mu+\nu$ denotes the realvalued function on $\downarrow V$ defined by

$$
(\mu+\nu)\left(V^{\prime}\right):=\mu\left(V^{\prime}\right)+\nu\left(V^{\prime}\right)
$$

for all $V^{\prime} \subseteq V$.

3. The real numbers, $\mathbb{R}$, form a ring, and so it is natural to see if a multiplicative structure can be put on $\Gamma \underline{\mathbb{R}^{\succeq}}$. The obvious 'definition' would be, for all $V$,

$$
(\mu \nu)(V):=\mu(V) \nu(V)
$$

for $\mu, \nu \in \Gamma \underline{\mathbb{R}^{\succeq}}$. However, this fails because the right hand side of (3.56) may not be order-reversing. This problem arises if $\mu(V)$ and $\nu(V)$ become negative: then, as $V$ gets smaller, these numbers get closer and closer to 0 , and then their product is a function that is order-preserving.

\footnotetext{
${ }^{16}$ Analogous arguments apply to the presheaves $\underline{\mathbb{R}} \underline{r}$ and $\underline{\mathbb{R}} \leftrightarrow$.
} 


\subsection{The Representation of Propositions From Pullbacks}

In paper I [2], we introduced a simple propositional language, $\mathcal{P} \mathcal{L}(S)$, for each system $S$, and discussed its representations for the case of classical physics. Then, in [3] we analysed the, far more complicated, quantum-theoretical representation of this language in the set of clopen subsets of the spectral presheaf, $\underline{\Sigma}$, in the topos $\operatorname{Sets}{ }^{\mathcal{V}(\mathcal{H})^{\mathrm{op}}}$. This gives a representation of the primitive propositions " $A \varepsilon \Delta$ " as

$$
\pi_{\mathrm{qt}}(A \varepsilon \Delta):=\delta^{o}(\hat{E}[A \in \Delta])
$$

where ' $\delta$ ' is the (outer) daseinisation operation, and $\hat{E}[A \in \Delta]$ is the spectral projection on the subset $\Delta \cap \operatorname{sp}(\hat{A})$ of the spectrum, $\operatorname{sp}(\hat{A})$, of the self-adjoint operator $\hat{A}$.

We now want to remark briefly on the nature, and representation, of propositions using the 'local' language $\mathcal{L}(S)$.

In any classical representation, $\sigma$, of $\mathcal{L}(S)$ in Sets, the representation, $\mathcal{R}_{\sigma}$, of the quantity-value symbol $\mathcal{R}$ is always just the real numbers $\mathbb{R}$. Therefore, it is simple to take a subset $\Delta \subseteq \mathbb{R}$ of $\mathbb{R}$, and construct the propositions " $A \varepsilon \Delta$ ". In fact, if $A_{\sigma}: \Sigma_{\sigma} \rightarrow \mathbb{R}$ is the representation of the function symbol $A$ with signature $\Sigma \rightarrow \mathcal{R}$, then $A_{\sigma}^{-1}(\Delta)$ is a subset of the symplectic manifold $\Sigma_{\sigma}$ (the representation of the ground type $\Sigma$ ). This subset, $A_{\sigma}^{-1}(\Delta) \subseteq \Sigma_{\sigma}$, represents the proposition " $A \varepsilon \Delta$ " in the Boolean algebra of all (Borel) subsets of $\Sigma_{\sigma}$.

We should consider the analogue of these steps in the representation, $\phi$, of the same language, $\mathcal{L}(S)$, in the topos $\tau_{\phi}:=\operatorname{Sets}^{\mathcal{V}(\mathcal{H})^{\mathrm{op}}}$. In fact, the issues to be discussed apply to a representation in any topos.

We first note that if $\Xi$ is a sub-object of $\mathcal{R}_{\phi}$, and if $A_{\phi}: \Sigma_{\phi} \rightarrow \mathcal{R}_{\phi}$, then there is an associated sub-object of $\Sigma_{\phi}$, denoted $A_{\phi}^{-1}(\Xi)$. Specifically, if $\chi_{\Xi}: \mathcal{R}_{\phi} \rightarrow \Omega_{\tau_{\phi}}$ is the characteristic arrow of the sub-object $\Xi$, then $A_{\phi}^{-1}(\Xi)$ is defined to be the sub-object of $\Sigma_{\phi}$ whose characteristic arrow is $\chi_{\Xi} \circ A_{\phi}: \Sigma_{\phi} \rightarrow \Omega_{\tau_{\phi}}$. These sub-objects are analogues of the subsets, $A_{\sigma}^{-1}(\Delta)$, of the classical state space $\Sigma_{\sigma}$ : as such, they can represent propositions. In this spirit, we could denote by " $A \varepsilon \Xi$ " the proposition which the sub-object $A_{\phi}^{-1}(\Xi)$ represents, although, of course, it would be a mistake to interpret " $A \varepsilon \Xi "$ as asserting that the value of something lies in something else: in a general topos, there are no such values.

In the case of quantum theory, the arrows $A_{\phi}: \Sigma_{\phi} \rightarrow \mathcal{R}_{\phi}$ are of the form $\breve{\delta}^{o}(A)$ : $\underline{\Sigma} \rightarrow \underline{\mathbb{R}^{\succeq}}$ where $\mathcal{R}_{\phi}:=\underline{\mathbb{R}^{\succeq}} \underline{17}$ It follows that the propositions in our $\mathcal{L}(S)$-theory are represented by the sub-objects $\breve{\delta}^{o}(A)^{-1}(\underline{\Xi})$ of $\underline{\Sigma}$, where $\underline{\Xi}$ is a sub-object of $\underline{\mathbb{R}^{\succeq}}$.

To interpret such propositions, note first that in the $\mathcal{P} \mathcal{L}(S)$-propositions " $A \varepsilon \Delta$ ", the range ' $\Delta$ ' belongs to the world that is external to the language. Consequently, the meaning of $\Delta$ is given independently of $\mathcal{P} \mathcal{L}(S)$. This 'externally interpreted' $\Delta$

\footnotetext{
${ }^{17}$ We can also use the other presheaves defined in Subsection $\underline{3.2}, \underline{\mathbb{R}} \preceq$ and $\underline{\mathbb{R}} \leftrightarrow$, as quantity-value objects and pull back their sub-objects to sub-objects of $\underline{\Sigma}$.
} 
is then inserted into the quantum representation of $\mathcal{P} \mathcal{L}(S)$ via the daseinisation of propositions discussed in paper II.

However, the situation is very different for the $\mathcal{L}(S)$-propositions " $A \varepsilon \Xi$ ". Here, the quantity ' $\Xi$ ' belongs to the particular topos $\tau_{\phi}$, and hence it is representation dependent. The implication is that the "meaning' of " $A \varepsilon \Xi$ " can only be discussed from 'within the topos' using the internal language that is associated with $\tau_{\phi}$, which, we recall, carries the translation of $\mathcal{L}(S)$ given by the topos-representation $\phi$.

From a conceptual perspective, this situation is 'relational', with the meanings of the various propositions being determined by their relations to each other as formulated in the internal language of the topos. Concomitantly, the meaning of 'truth' cannot be understood using the correspondence theory (much favoured by instrumentalists) for there is nothing external to which a proposition can 'correspond'. Instead, what is needed is more like a coherence theory of truth in which a whole body of propositions is considered together [18]. This is a fascinating subject, but further discussion must be deferred to later work.

\section{The Presheaf $k\left(\underline{\mathbb{R}^{\succeq}}\right)$}

\subsection{Some Background Information}

\subsubsection{Preliminary Remarks}

We have shown how each self-adjoint operator, $\hat{A}$, on the Hilbert space $\mathcal{H}$ gives rise to an arrow $\breve{\delta}^{o}(A): \underline{\Sigma} \rightarrow \underline{\mathbb{R}^{\succeq}}$ in the topos $\operatorname{Sets}^{\mathcal{V}(\mathcal{H})^{\text {op }}}$. Thus, in the topos representation, $\phi$, of $\mathcal{L}(S)$ for the theory-type 'quantum theory', the arrow $\breve{\delta}^{o}(A): \underline{\Sigma} \rightarrow \underline{\mathbb{R}^{\succeq}}$ is one possible choice 18 for the representation, $A_{\phi}: \Sigma_{\phi} \rightarrow \mathcal{R}_{\phi}$, of the function symbol, $A: \Sigma \rightarrow \mathcal{R}$.

This implies that the quantity-value object, $\mathcal{R}_{\phi}$, is the presheaf, $\underline{\mathbb{R}} \succeq$. However, although such an identification is possible, it does impose certain restrictions on the formalism. These stem from the fact that $\underline{\mathbb{R}^{\succeq}}$ is only a monoid-object in $\operatorname{Sets}{ }^{\mathcal{V}(\mathcal{H})^{\mathrm{op}}}$, and $\Gamma \underline{\mathbb{R}^{\succeq}}$ is only a monoid, whereas the real numbers of standard physics are an abelian group; indeed, they are a commutative ring.

In standard classical physics, $\operatorname{Hom}_{\text {Sets }}\left(\Sigma_{\sigma}, \mathbb{R}\right)$ is the set of real-valued functions on the manifold $\Sigma_{\sigma}$; as such, it possesses the structure of a commutative ring. On the other hand, the set of arrows $\operatorname{Hom}_{\operatorname{Sets}} \mathcal{V}(\mathcal{H})^{\text {op }}(\underline{\Sigma}, \underline{\mathbb{R}} \succeq)$ has only the structure of an additive monoid. This additive structure is defined locally in the obvious way: i.e., if $\alpha, \beta \in \operatorname{Hom}_{\operatorname{Sets}^{\mathcal{V}}(\mathcal{H})^{\text {op }}}\left(\underline{\Sigma}, \underline{\mathbb{R}^{\succeq}}\right)$ we define, for all stages $V \in \mathrm{Ob}(\mathcal{V}(\mathcal{H}))$, and all $\lambda \in \underline{\Sigma}_{V}$, (c.f. $(3.55))$

$$
(\alpha+\beta)_{V}(\lambda):=\alpha_{V}(\lambda)+\beta_{V}(\lambda)
$$

where both sides of (4.1) are elements of $\underline{\mathbb{R}}_{V}^{\succeq}$, i.e., order-reversing functions from $\downarrow V$ to $\mathbb{R}$. It is clear that the right hand side of (4.1) is an order-reversing function, so that

\footnotetext{
${ }^{18}$ Another choice is to use the presheaf $\underline{\mathbb{R} \leftrightarrow}$ as the quantity-value object.
} 
$\alpha+\beta$ is well defined 19

Arguably, the fact that $\operatorname{Hom}_{\operatorname{Sets}^{\mathcal{V}(\mathcal{H})^{\text {op }}}}\left(\underline{\Sigma}, \underline{\mathbb{R}^{\succeq}}\right)$ is only a monoid 20 is a weakness in so far as we are trying to make quantum theory 'look' as much like classical physics as possible. Of course, in more obscure applications such as Planck-length quantum gravity, the nature of the quantity-value object is very much open for debate. But when applied to regular physics, we might like our formalism to look more like classical physics than the monoid-only structure of $\operatorname{Hom}_{\operatorname{Sets}^{\mathcal{V}}(\mathcal{H})^{\text {op }}}(\underline{\Sigma}, \underline{\mathbb{R}} \underline{\underline{ }})$.

There are also more practical reasons for wanting to extend our current formalism. For example, given the arrows $\breve{\delta}^{o}\left(A^{2}\right)$ and $\breve{\delta}^{o}(A)$, it would be interesting to define an intrinsic dispersion 21 .

$$
\nabla(\hat{A}):=\breve{\delta}^{o}\left(A^{2}\right)-\breve{\delta}^{o}(A)^{2}
$$

but this is meaningless because (i) the square, $\breve{\delta}^{o}(A)^{2}$, of the arrow $\breve{\delta}^{o}(A): \underline{\Sigma} \rightarrow \underline{\mathbb{R}} \succeq$ is not well-defined; and (ii) even if it was, the difference between two arrows from $\underline{\Sigma}$ to $\underline{\mathbb{R}^{\succeq}}$ is not well-defined. Thus we need to be able to take the square of an element in $\overline{\operatorname{Hom}}_{\text {Sets }} \mathcal{v}(\mathcal{H})^{\text {op }}(\underline{\Sigma}, \underline{\mathbb{R}} \succeq)$, and to subtract such arrows.

The need for a subtraction, i.e. some sort of abelian group structure on $\underline{\mathbb{R}} \succeq$, brings to mind the well-known Grothendieck $k$-construction that is much used in algebraic topology and other branches of pure mathematics. This gives a way of 'extending' an abelian semi-group to become an abelian group, and we want to see if this technique can be adapted to the present situation. The goal is to construct a 'Grothendieck completion', $k\left(\underline{\mathbb{R}}\right.$ ) , of $\underline{\mathbb{R}^{\succeq}}$ that is an abelian-group object in the topos $\operatorname{Sets}{ }^{\mathcal{V}}(\mathcal{H})^{\text {op }}$.

Of course, what we want is more, namely a well-defined operation of taking squares. We will see that, in a limited sense, this is obtained at no extra cost.22

\subsubsection{The Grothendieck $k$-Construction for a Semi-Group}

Let us briefly review the Grothendieck construction for an abelian monoid $M$.

Definition 4.1 $A$ group completion of $M$ is an abelian group $k(M)$ together with a monoid map $\theta: M \rightarrow k(M)$ that is universal. Namely, given any monoid morphism $\phi: M \rightarrow G$, where $G$ is an abelian group, there exists a unique group morphism $\phi^{\prime}: k(M) \rightarrow G$ such that $\phi$ factors through $\phi^{\prime} ;$ i.e., we have the commutative diagram

\footnotetext{
${ }^{19}$ To avoid confusion we should emphasise that, in general, the sum $\delta^{o}(\hat{A})+\delta^{o}(\hat{B})$ is not equal to $\delta^{o}(\hat{A}+\hat{B})$.

${ }^{20}$ An internal version of this result would show that the exponential object $\underline{\mathbb{R}} \underline{\underline{\Sigma}}$ is a monoid object in the topos $\operatorname{Sets}^{\mathcal{V}(\mathcal{H})^{\text {op }}}$. This could well be true, but we have not studied it in detail.

${ }^{21}$ The notation used here is potentially a little misleading. We have not given any meaning to ' $A^{2}$, in the language $\mathcal{L}(S)$; i.e., in its current form, the language does not give meaning to the square of a function symbol. Therefore, when we write $\breve{\delta}^{o}\left(A^{2}\right)$ this must be understood as being the Gel'fand transform of the outer daseinisation of the operator $\hat{A}^{2}$.

${ }^{22}$ Ideally, we might like $k\left(\underline{\mathbb{R}^{\succeq}}\right)$ to be a commutative-ring object, but this is not true.
} 


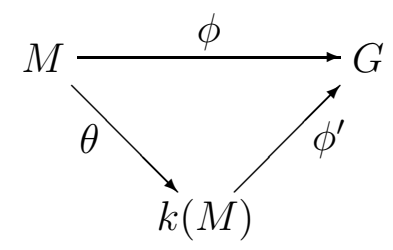

with $\phi=\phi^{\prime} \circ \theta$.

It is easy to see that any such $k(M)$ is unique up to isomorphism.

To prove existence, first take the set of all pairs $(a, b) \in M \times M$, each of which is to be thought of heuristically as $a-b$. Then, note that if inverses existed in $M$, we would have $a-b=c-d$ if and only if $a+d=c+b$. This suggests defining an equivalence relation on $M \times M$ in the following way:

$$
(a, b) \equiv(c, d) \text { iff } \exists g \in M \text { such that } a+d+g=b+c+g \text {. }
$$

Definition 4.2 The Grothendieck completion of a monoid $M$ is the pair $(k(M), \theta)$ defined as follows:

(i) $k(M)$ is the set of equivalence classes $[a, b]$, where the equivalence relation is defined in (4.3). A group law on $k(M)$ is defined by

$$
\begin{aligned}
& \text { (i) }[a, b]+[c, d]:=[a+c, b+d] \text {, } \\
& \text { (ii) } 0_{k(M)}:=\left[0_{M}, 0_{M}\right], \\
& \text { (iii) }-[a, b]:=[b, a],
\end{aligned}
$$

where $0_{M}$ is the unit in the abelian monoid $M$.

(ii) The $\operatorname{map} \theta: M \rightarrow k(M)$ is defined by

$$
\theta(a):=[a, 0]
$$

for all $a \in M$.

It is straightforward to show that (i) these definitions are independent of the representative elements in the equivalence classes; (ii) the axioms for a group are satisfied; and (iii) the map $\theta$ is universal in the sense mentioned above.

It is also clear that $k$ is a functor from the category of abelian monoids to the category of abelian groups. For, if $f: M_{1} \rightarrow M_{2}$ is a morphism between abelian monoids, define $k(f): k\left(M_{1}\right) \rightarrow k\left(M_{2}\right)$ by $k(f)[a, b]:=[f(a), f(b)]$ for all $a, b \in M_{1}$.

\subsection{Functions of Bounded Variation and $\Gamma \underline{\mathbb{R}^{\succeq}}$}

These techniques will now be applied to the set, $\Gamma \underline{\mathbb{R}} \succeq$, of global elements of $\underline{\mathbb{R}} \succeq$. As was discussed in Section [3.2, global elements of $\underline{\mathbb{R}} \succeq$ are in one-to-one correspondence with 
order-reversing functions on the category $\mathcal{V}(\mathcal{H})$; i.e., with functions $\mu: \operatorname{Ob}(\mathcal{V}(\mathcal{H})) \rightarrow \mathbb{R}$ such that, for all $V_{1}, V_{2} \in \mathrm{Ob}(\mathcal{V}(\mathcal{H}))$, if $V_{2} \subseteq V_{1}$ then $\mu\left(V_{2}\right) \geq \mu\left(V_{1}\right)$; see (3.53). The monoid law is given by (3.5.5).

Since $\Gamma \underline{\mathbb{R}^{\succeq}}$ is an abelian monoid, the Grothendieck construction can be applied to give an abelian group $k(\Gamma \underline{\mathbb{R}} \succeq)$. This is defined to be the set of equivalence classes $[\lambda, \kappa]$ where $\lambda, \kappa \in \Gamma \underline{\mathbb{R}^{\succeq}}$, and where $\left(\lambda_{1}, \kappa_{1}\right) \equiv\left(\lambda_{2}, \kappa_{2}\right)$ if, and only if, there exists $\alpha \in \Gamma \underline{\mathbb{R}^{\succeq}}$, such that

$$
\lambda_{1}+\kappa_{2}+\alpha=\kappa_{1}+\lambda_{2}+\alpha
$$

Intuitively, we can think of $[\lambda, \kappa]$ as being ' $\lambda-\kappa$ ', and embed $\Gamma \underline{\mathbb{R}^{\succeq}}$ in $k\left(\Gamma \underline{\mathbb{R}^{\succeq}}\right)$ by $\lambda \mapsto[\lambda, 0]$. However, $\lambda, \kappa$ are $\mathbb{R}$-valued functions on $\operatorname{Ob}(\mathcal{V}(\mathcal{H}))$ and hence, in this case, the expression ' $\lambda-\kappa$ ' also has a literal meaning: i.e., as the function $(\lambda-\kappa)(V):=$ $\lambda(V)-\kappa(V)$ for all $V \in \mathrm{Ob}(\mathcal{V}(\mathcal{H}))$.

This is not just a coincidence of notation. Indeed, let $F(\operatorname{Ob}(\mathcal{V}(\mathcal{H})), \mathbb{R})$ denote the set of all real-valued functions on $\operatorname{Ob}(\mathcal{V}(\mathcal{H}))$. Then we can construct the map,

$$
\begin{aligned}
j: k\left(\Gamma \underline{\mathbb{R}^{\succeq}}\right) & \rightarrow F(\mathrm{Ob}(\mathcal{V}(\mathcal{H})), \mathbb{R}) \\
{[\lambda, \kappa] } & \mapsto \lambda-\kappa
\end{aligned}
$$

which is well-defined on equivalence classes.

It is easy to see that the map in (4.9) is injective. This raises the question of the image in $F(\mathrm{Ob}(\mathcal{V}(\mathcal{H})), \mathbb{R})$ of the map $j$ : i.e., what types of real-valued function on $\operatorname{Ob}(\mathcal{V}(\mathcal{H}))$ can be written as the difference between two order-reversing functions?

For functions $f: \mathbb{R} \rightarrow \mathbb{R}$, it is a standard result that a function can be written as the difference between two monotonic functions if, and only if, it has bounded variation. The natural conjecture is that a similar result applies here. To show this, we proceed as follows.

Let $f: \operatorname{Ob}(\mathcal{V}(\mathcal{H})) \rightarrow \mathbb{R}$ be a real-valued function on the set of objects in the category $\mathcal{V}(\mathcal{H})$. At each $V \in \operatorname{Ob}(\mathcal{V}(\mathcal{H}))$, consider a finite chain

$$
C:=\left\{V_{0}, V_{1}, V_{2}, \ldots, V_{n-1}, V \mid V_{0} \subset V_{1} \subset V_{2} \subset \cdots \subset V_{n-1} \subset V\right\}
$$

of proper subsets, and define the variation of $f$ on this chain to be

$$
V_{f}(C):=\sum_{j=1}^{n}\left|f\left(V_{j}\right)-f\left(V_{j-1}\right)\right|
$$

where we set $V_{n}:=V$. Now take the supremum of $V_{f}(C)$ for all such chains $C$. If this is finite, we say that $f$ has a bounded variation and define

$$
I_{f}(V):=\sup _{C} V_{f}(C)
$$

Then it is clear that (i) $V \mapsto I_{f}(V)$ is an order-preserving function on $\operatorname{Ob}(\mathcal{V}(\mathcal{H}))$; (ii) $f-I_{f}$ is an order-reversing function on $\mathrm{Ob}\left(\mathcal{V}(\mathcal{H})\right.$ ); and (iii) $-I_{f}$ is an order-reversing 
function on $\operatorname{Ob}(\mathcal{V}(\mathcal{H}))$. Thus, any function, $f$, of bounded variation can be written as

$$
f \equiv\left(f-I_{f}\right)-\left(-I_{f}\right)
$$

which is the difference of two order-reversing functions; i.e., $f$ can be expressed as the difference of two elements of $\Gamma \underline{\mathbb{R}}$.

Conversely, it is a straightforward modification of the proof for functions $f: \mathbb{R} \rightarrow \mathbb{R}$, to show that if $f: \operatorname{Ob}(\mathcal{V}(\mathcal{H})) \rightarrow \mathbb{R}$ is the difference of two order-reversing functions, then $f$ is of bounded variation. The conclusion is that $k(\Gamma \underline{\mathbb{R}} \succeq)$ is in bijective correspondence with the set, $\operatorname{BV}(\mathrm{Ob}(\mathcal{V}(\mathcal{H})), \mathbb{R})$, of functions $f: \operatorname{Ob}(\mathcal{V}(\mathcal{H})) \rightarrow \mathbb{R}$ of bounded variation.

\subsection{Taking Squares in $k\left(\Gamma \underline{\mathbb{R}^{\succeq}}\right)$.}

We can now think of $k(\Gamma \underline{\mathbb{R}} \succeq)$ in two ways: (i) as the set of equivalence classes $[\lambda, \kappa]$, of elements $\lambda, \kappa \in \Gamma \underline{\mathbb{R}^{\succeq}}$; and (ii) as the set, $\operatorname{BV}(\mathrm{Ob}(\mathcal{V}(\mathcal{H})), \mathbb{R})$, of differences $\lambda-\kappa$ of such elements.

As expected, $\mathrm{BV}(\mathrm{Ob}(\mathcal{V}(\mathcal{H})), \mathbb{R})$ is an abelian group. Indeed: suppose $\alpha=\lambda_{1}-\kappa_{1}$ and $\beta=\lambda_{2}-\kappa_{2}$ with $\lambda_{1}, \lambda_{2}, \kappa_{1}, \kappa_{2} \in \Gamma \underline{\mathbb{R}^{\succeq}}$, then

$$
\alpha+\beta=\left(\lambda_{1}+\lambda_{2}\right)-\left(\kappa_{1}+\kappa_{2}\right)
$$

Hence $\alpha+\beta$ belongs to $\mathrm{BV}(\mathrm{Ob}(\mathcal{V}(\mathcal{H})), \mathbb{R})$ since $\lambda_{1}+\lambda_{2}$ and $\kappa_{1}+\kappa_{2}$ belong to $\Gamma \underline{\mathbb{R}} \succeq$.

The definition of $[\lambda, 0]^{2}$. We will now show how to take the square of elements of $k\left(\Gamma \underline{\mathbb{R}^{\succeq}}\right)$ that are of the form $[\lambda, 0]$. Clearly, $\lambda^{2}$ is well-defined as a function on $\operatorname{Ob}(\mathcal{V}(\mathcal{H}))$, but it may not belong to $\Gamma \underline{\mathbb{R}^{\succeq}}$. Indeed, if $\lambda(V)<0$ for any $V$, then the function $V \mapsto \lambda^{2}(V)$ can get smaller as $V$ gets smaller, so it is order-preserving instead of order-reversing.

This suggests the following strategy. First, define functions $\lambda_{+}$and $\lambda_{-}$by

$$
\lambda_{+}(V):= \begin{cases}\lambda(V) & \text { if } \lambda(V) \geq 0 \\ 0 & \text { if } \lambda(V)<0\end{cases}
$$

and

$$
\lambda_{-}(V):= \begin{cases}0 & \text { if } \lambda(V) \geq 0 \\ \lambda(V) & \text { if } \lambda(V)<0 .\end{cases}
$$

Clearly, $\lambda(V)=\lambda_{+}(V)+\lambda_{-}(V)$ for all $V \in \mathrm{Ob}(\mathcal{V}(\mathcal{H}))$. Also, for all $V, \lambda_{+}(V) \lambda_{-}(V)=$ 0 , and hence

$$
\lambda(V)^{2}=\lambda_{+}(V)^{2}+\lambda_{-}(V)^{2}
$$

However, (i) the function $V \mapsto \lambda_{+}(V)^{2}$ is order-reversing; and (ii) the function $V \mapsto$ $\lambda_{-}(V)^{2}$ is order-preserving. But then $V \mapsto-\lambda_{-}(V)^{2}$ is order-reversing. Hence, by rewriting (4.17) as

$$
\lambda(V)^{2}=\lambda_{+}(V)^{2}-\left(-\lambda_{-}(V)^{2}\right)
$$


we see that the function $V \mapsto \lambda^{2}(V):=\lambda(V)^{2}$ is an element of $\mathrm{BV}(\mathrm{Ob}(\mathcal{V}(\mathcal{H})), \mathbb{R})$.

In terms of $k(\Gamma \underline{\mathbb{R}} \succeq)$, we can define

$$
[\lambda, 0]^{2}:=\left[\lambda_{+}^{2},-\lambda_{-}^{2}\right]
$$

which belongs to $k(\Gamma \underline{\mathbb{R}} \succeq)$. Hence, although there exist $\lambda \in \Gamma \underline{\mathbb{R}} \succeq$ that have no square in $\Gamma \underline{\mathbb{R}} \succeq$, such global elements of $\underline{\mathbb{R}^{\succeq}} d o$ have squares in the $k$-completion, $k(\Gamma \underline{\mathbb{R}} \succeq)$. On the level of functions of bounded variation, we have shown that the square of a monotonic (order-reversing) function is a function of bounded variation.

On the other hand, we cannot take the square of an arbitrary element $[\lambda, \kappa] \in \Gamma \underline{\mathbb{R}^{\succeq}}$, since the square of a function of bounded variation need not be a function of bounded variation 23

\subsection{The Object $k\left(\underline{\mathbb{R}^{\succeq}}\right)$ in the Topos $\operatorname{Sets}^{\mathcal{V}(\mathcal{H})^{\mathrm{op}}}$}

\subsubsection{The Definition of $k\left(\underline{\mathbb{R}^{\succeq}}\right)$}

The next step is to translate these results about the set $k\left(\Gamma \underline{\mathbb{R}^{\succeq}}\right)$ into the construction of an object $k\left(\underline{\mathbb{R}^{\succeq}}\right)$ in the topos $\operatorname{Sets}^{\mathcal{V}(\mathcal{H})^{\mathrm{op}}}$. We anticipate that, if this can be done, then $k(\Gamma \underline{\mathbb{R}} \succeq) \simeq \Gamma k(\underline{\mathbb{R}} \succeq)$.

As was discussed in Section (3.2), the presheaf $\underline{\mathbb{R}^{\succeq}}$ is defined at each stage $V$ by

$$
\underline{\mathbb{R}}_{V}:=\{\lambda: \downarrow V \rightarrow \mathbb{R} \mid \lambda \in \mathcal{O} \mathcal{R}(\downarrow V, \mathbb{R})\} .
$$

If $i_{V^{\prime} V}: V^{\prime} \subseteq V$, then the presheaf map from $\underline{\mathbb{R}}_{V}$ to $\underline{\mathbb{R}}_{V^{\prime}}$ is just the restriction of the order-reversing functions from $\downarrow V$ to $\downarrow V^{\prime}$.

The first step in constructing $k\left(\underline{\mathbb{R}^{\succeq}}\right)$ is to define an equivalence relation on pairs of functions, $\lambda, \kappa \in \underline{\mathbb{R}}^{\succeq}$, for each stage $V$, by saying that $\left(\lambda_{1}, \kappa_{1}\right) \equiv\left(\lambda_{2}, \kappa_{2}\right)$ if, and only, there exists $\alpha \in \underline{\mathbb{R}}_{V}$ such that

$$
\lambda_{1}\left(V^{\prime}\right)+\kappa_{2}\left(V^{\prime}\right)+\alpha\left(V^{\prime}\right)=\kappa_{1}\left(V^{\prime}\right)+\lambda_{2}\left(V^{\prime}\right)+\alpha\left(V^{\prime}\right)
$$

for all $V^{\prime} \subseteq V$

Definition 4.3 The presheaf $k(\underline{\mathbb{R}} \succeq)$ is defined over the category $\mathcal{V}(\mathcal{H})$ in the following way.

(i) On objects $V \in \mathrm{Ob}(\mathcal{V}(\mathcal{H}))$ :

$$
k(\underline{\mathbb{R}} \succeq)_{V}:=\{[\lambda, \kappa] \mid \lambda, \kappa \in \mathcal{O} \mathcal{R}(\downarrow V, \mathbb{R})\},
$$

where $[\lambda, \kappa]$ denotes the $k$-equivalence class of $(\lambda, \kappa)$.

\footnotetext{
${ }^{23}$ We have to consider functions like $\left(\lambda_{+}+\lambda_{-}-\left(\kappa_{+}+\kappa_{-}\right)\right)^{2}$, which contains terms of the form $\lambda_{+} \kappa_{-}$and $\lambda_{-} \kappa_{+}$: in general, these are neither order-preserving nor order-reversing.
} 
(ii) On morphisms $i_{V^{\prime} V}: V^{\prime} \subseteq V$ : The arrow $k\left(\underline{\mathbb{R}^{\succeq}}\right)\left(i_{V^{\prime} V}\right): k\left(\underline{\mathbb{R}^{\succeq}}\right)_{V} \rightarrow k\left(\underline{\mathbb{R}^{\succeq}}\right)_{V^{\prime}}$ is given by $\left(k\left(\underline{\mathbb{R}^{\succeq}}\right)\left(i_{V^{\prime} V}\right)\right)([\lambda, \kappa]):=\left[\left.\lambda\right|_{V^{\prime}},\left.\kappa\right|_{V^{\prime}}\right]$ for all $[\lambda, \kappa] \in k(\underline{\mathbb{R}} \succeq)_{V}$.

It is straightforward to show that $k\left(\underline{\mathbb{R}^{\succeq}}\right)$ is an abelian group-object in the topos

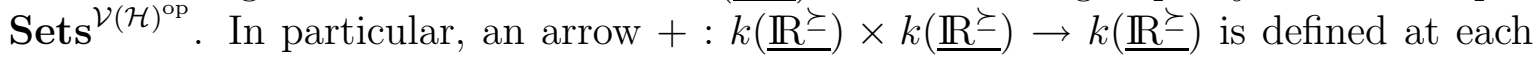
stage $V$ by

$$
+_{V}\left(\left[\lambda_{1}, \kappa_{1}\right],\left[\lambda_{2}, \kappa_{2}\right]\right):=\left[\lambda_{1}+\lambda_{2}, \kappa_{1}+\kappa_{2}\right]
$$

for all $\left(\left[\lambda_{1}, \kappa_{1}\right],\left[\lambda_{2}, \kappa_{2}\right]\right) \in k\left(\underline{\mathbb{R}^{\succeq}}\right)_{V} \times k\left(\underline{\mathbb{R}^{\succeq}}\right)_{V}$. It is easy to see that (i) $\Gamma k\left(\underline{\mathbb{R}^{\succeq}}\right) \simeq$ $k\left(\Gamma \underline{\mathbb{R}^{\succeq}}\right)$; and (ii) $\underline{\mathbb{R}^{\succeq}}$ is a sub-object of $k\left(\underline{\mathbb{R}^{\succeq}}\right)$ in the topos $\operatorname{Sets}^{\mathcal{V}(\mathcal{H})^{\mathrm{op}}}$.

\subsubsection{The Presheaf $k\left(\underline{\mathbb{R}^{\succeq}}\right)$ as the Quantity-Value Object.}

We can now identify $k\left(\underline{\mathbb{R}^{\succeq}}\right)$ as a possible quantity-value object in $\operatorname{Sets}^{\mathcal{V}(\mathcal{H})^{\mathrm{op}}}$. To each bounded, self-adjoint operator $\hat{A}$, there is an arrow $\left[\breve{\delta}^{o}(A)\right]: \underline{\Sigma} \rightarrow k\left(\underline{\mathbb{R}}^{\succeq}\right)$, given by first sending $\hat{A} \in \mathcal{B}(\mathcal{H})_{\mathrm{sa}}$ to $\breve{\delta}^{o}(A)$ and then taking $k$-equivalence classes. More precisely, one takes the monic $\iota: \underline{\mathbb{R}^{\succeq}} \hookrightarrow k\left(\underline{\mathbb{R}^{\succeq}}\right)$ and then constructs $\iota \circ \breve{\delta}^{o}(A): \underline{\Sigma} \rightarrow k\left(\underline{\mathbb{R}^{\succeq}}\right)$.

Since, for each stage $V$, the elements in the image of $\left[\breve{\delta}^{o}(A)\right]_{V}=\left(\iota \circ \breve{\delta}^{o}(A)\right)_{V}$ are of the form $[\lambda, 0], \lambda \in \underline{\mathbb{R}}_{V}^{\succeq}$, their square is well-defined. From a physical perspective, the use of $k(\underline{\mathbb{R}} \succeq)$ rather than $\underline{\mathbb{R}^{\succeq}}$ renders possible the definition of things like the 'intrinsic dispersion', $\nabla(\hat{A}):=\breve{\delta}^{o}\left(A^{2}\right)-\breve{\delta}^{o}(A)^{2}$; see (4.2).

The relation between $\underline{\mathbb{R}^{\leftrightarrow}}$ and $k\left(\underline{\mathbb{R}^{\succeq}}\right)$. In Section 3.2 , we considered the presheaf $\underline{\mathbb{R}^{\leftrightarrow}}$ of order-preserving and order-reversing functions as a possible quantity-value object. The advantage of this presheaf is the symmetric utilisation of inner and outer daseinisation, and the associated physical interpretation of arrows from $\underline{\Sigma}$ to $\underline{\mathbb{R}} \leftrightarrow$.

It transpires that $\underline{\mathbb{R}^{\leftrightarrow}}$ is closely related to $k\left(\underline{\mathbb{R}^{\succeq}}\right)$. Namely, for each $V$, we define an equivalence relation $\equiv$ on $\underline{\mathbb{R}}^{\leftrightarrow} V$ by

$$
\left(\mu_{1}, \nu_{1}\right) \equiv\left(\mu_{2}, \nu_{2}\right) \text { iff } \mu_{1}+\nu_{1}=\mu_{2}+\nu_{2} .
$$

Then $\underline{\mathbb{R}^{\leftrightarrow}} / \equiv$ is isomorphic to $k\left(\underline{\mathbb{R}^{\succeq}}\right)$ under the mapping

$$
[\mu, \nu] \mapsto[\nu,-\mu] \in k\left(\underline{\mathbb{R}^{\succeq}}\right)_{V}
$$

for all $V$ and all $[\mu, \nu] \in\left(\underline{\mathbb{R}^{\leftrightarrow}} / \equiv\right)_{V}$. (Recall that $[\mu, \nu] \in\left(\underline{\mathbb{R}^{\leftrightarrow}} / \equiv\right)_{V}$ means that $\mu$ is order-preserving and $\nu$ is order-reversing.)

However, there is a difference between the arrows that represent physical quantities. The arrow $\left[\breve{\delta}^{o}(A)\right]: \underline{\Sigma} \rightarrow k(\underline{\mathbb{R}} \succeq)$ is given by first sending $\hat{A} \in \mathcal{B}(\mathcal{H})_{\text {sa }}$ to $\breve{\delta}^{o}(A)$ and then taking $k$-equivalence classes - a construction that only involves outer daseinisation. On the other hand, there is an arrow $[\breve{\delta}(A)]: \underline{\Sigma} \rightarrow \underline{\mathbb{R} \leftrightarrow} / \equiv$, given by first sending $\hat{A}$ to $\breve{\delta}(A)$ and then taking the equivalence classes defined in (4.24). This involves both inner and outer daseinisation.

We can show that $\left[\breve{\delta}^{o}(A)\right]$ uniquely determines $\hat{A}$ (see Appendix), but currently it is an open question if $[\breve{\delta}(A)]$ also fixes $\hat{A}$ uniquely. 


\section{The Role of Unitary Operators}

\subsection{The Daseinisation of Unitary Operators}

Unitary operators play an important role in the formulation of quantum theory, and we need to understand the analogue of this in our topos formalism.

Unitary operators arise in the context of both 'covariance' and 'invariance'. In elementary quantum theory, the 'covariance' aspect comes the fact that if we have made the associations

$$
\begin{aligned}
\text { Physical state } & \mapsto \text { state vector }|\psi\rangle \in \mathcal{H} \\
\text { Physical observable } A & \mapsto \text { self-adjoint operator } \hat{A} \text { acting on } \mathcal{H}
\end{aligned}
$$

then the same physical predictions will be obtained if the following associations are used instead

$$
\begin{aligned}
\text { Physical state } & \mapsto \text { state vector } \hat{U}|\psi\rangle \in \mathcal{H} \\
\text { Physical observable } A & \mapsto \text { self-adjoint operator } \hat{U} \hat{A} \hat{U}^{-1} \text { acting on } \mathcal{H}
\end{aligned}
$$

for any unitary operator $\hat{U}$. Thus the mathematical representatives of physical quantities are defined only up to arbitrary transformations of the type above. In nonrelativistic quantum theory, this leads to the canonical commutation relations; the angular-momentum commutator algebra; and the unitary time displacement operator. Similar considerations in relativistic quantum theory involve the Poincare group.

The 'invariance' aspect of unitary operators arises when the operator commutes with the Hamiltonian, giving rise to conserved quantities.

Daseinisation of unitary operators. As a side remark, we first consider the question if daseinisation can be applied to a unitary operator $\hat{U}$. The answer is clearly 'yes', via the spectral representation:

$$
\hat{U}=\int_{\mathbb{R}} e^{i \lambda} d \hat{E}_{\lambda}^{U}
$$

where $\lambda \mapsto E_{\lambda}^{\hat{U}}$ is the spectral family for $\hat{U}$. Then, in analogy with (2.15 2.16) we have the following:

Definition 5.1 The outer daseinisation, $\delta^{o}(\hat{U})$, resp. the inner daseinisation, $\delta^{i}(\hat{U})$, of a unitary operator $\hat{U}$ are defined as follows:

$$
\begin{aligned}
\delta^{o}(\hat{U})_{V} & :=\int_{\mathbb{R}} e^{i \lambda} d\left(\delta_{V}^{i}\left(\hat{E}_{\lambda}^{U}\right)\right), \\
\delta^{i}(\hat{U})_{V} & :=\int_{\mathbb{R}} e^{i \lambda} d\left(\bigwedge_{\mu>\lambda} \delta_{V}^{o}\left(\hat{E}_{\mu}^{U}\right)\right),
\end{aligned}
$$


at each stage $V$.

To interpret these entities 24 we need to introduce a new presheaf defined as follows.

Definition 5.2 The outer, unitary de Groote presheaf, $\underline{\underline{U}}$, is defined by:

(i) On objects $V \in \mathrm{Ob}(\mathcal{V}(\mathcal{H})): \underline{\mathbb{U}}_{V}:=V_{\mathrm{un}}$, the collection of unitary operators in $V$.

(ii) On morphisms $i_{V^{\prime} V}: V^{\prime} \subseteq V$ : The mapping $\underline{\mathbb{U}}\left(i_{V^{\prime} V}\right): \underline{\mathbb{U}}_{V} \rightarrow \underline{\mathbb{U}}_{V^{\prime}}$ is given by

$$
\begin{aligned}
\underline{\mathbb{U}}\left(i_{V^{\prime} V}\right)(\hat{\alpha}) & :=\delta^{o}(\hat{\alpha})_{V^{\prime}} \\
& =\int_{\mathbb{R}} e^{i \lambda} d\left(\delta^{i}\left(\hat{E}_{\lambda}^{\alpha}\right)_{V^{\prime}}\right) \\
& =\int_{\mathbb{R}} e^{i \lambda} d\left(\underline{I}\left(i_{V^{\prime} V}\right)\left(\hat{E}_{\lambda}^{\alpha}\right)\right)
\end{aligned}
$$

for all $\hat{\alpha} \in \underline{\mathbb{U}}_{V}$.

Clearly, (i) there is an analogous definition of an 'inner', unitary de Groote presheaf; and (ii) the map $V \mapsto \delta^{o}(\hat{U})_{V}$ defines a global element of $\underline{\mathbf{U}}$.

This definition has the interesting consequence that, at each stage $V$,

$$
\delta^{o}\left(e^{i \hat{A}}\right)_{V}=e^{i \delta^{o}(\hat{A})_{V}}
$$

A particular example of this construction is the one-parameter family of unitary operators, $t \mapsto e^{i t \hat{H}}$, where $\hat{H}$ is the Hamiltonian of the system.

Of course, in our case everything commutes. Thus suppose $g \mapsto \hat{U}_{g}$ is a representation of a Lie group $G$ on the Hilbert space $\mathcal{H}$. Then these operators can be daseinised to give the map $g \mapsto \delta^{o}\left(\hat{U}_{g}\right)$, but generally this is not a representation of $G$ (or of its Lie algebra) since, at each stage $V$ we have

$$
\delta^{o}\left(\hat{U}_{g_{1}}\right)_{V} \delta^{o}\left(\hat{U}_{g_{2}}\right)_{V}=\delta^{o}\left(\hat{U}_{g_{2}}\right)_{V} \delta^{o}\left(\hat{U}_{g_{1}}\right)_{V}
$$

for all $g_{1}, g_{2} \in G$. Clearly, there is an analogous result for inner daseinisation.

\subsection{Unitary Operators and Arrows in $\operatorname{Sets}^{\mathcal{V}(\mathcal{H})^{\mathrm{op}}}$.}

\subsubsection{The Definition of $\ell_{\hat{U}}: \mathrm{Ob}(\mathcal{V}(\mathcal{H})) \rightarrow \operatorname{Ob}(\mathcal{V}(\mathcal{H}))$}

In classical physics, the analogue of unitary operators are 'canonical transformations'; i.e., symplectic diffeomorphisms from the state space $\mathcal{S}$ to itself. This suggests that should try to associate arrows in $\operatorname{Sets}^{\mathcal{V}(\mathcal{H})^{\mathrm{op}}}$ with each unitary operator $\hat{U}$.

\footnotetext{
${ }^{24}$ It would be possible to 'complexify' the presheaf $k\left(\underline{\mathbb{R}^{\succeq}}\right)$ in order to represent unitary operators as arrows from $\underline{\Sigma}$ to $\mathbb{C} k(\underline{\mathbb{R}})$. However, there is no obvious physical use for this procedure.
} 
Thus we want to see if unitary operators can act on the objects in $\operatorname{Sets}^{\mathcal{V}(\mathcal{H})^{\mathrm{op}}}$. In fact, if $\mathcal{U}(\mathcal{H})$ denotes the group of all unitary operators in $\mathcal{H}$, we would like to find a realisation of $\mathcal{U}(\mathcal{H})$ in the topos $\operatorname{Sets}^{\mathcal{V}(\mathcal{H})^{\text {op }}}$.

As a first step, if $\hat{U} \in \mathcal{U}(\mathcal{H})$ and $V \in \operatorname{Ob}(\mathcal{V}(\mathcal{H}))$ is an abelian von Neumann subalgebra of $\mathcal{B}(\mathcal{H})$, let us define

$$
\ell_{\hat{U}}(V):=\left\{\hat{U} \hat{A} \hat{U}^{-1} \mid \hat{A} \in V\right\}
$$

It is clear that $\ell_{\hat{U}}(V)$ is a unital, abelian algebra of operators, and that self-adjoint operators are mapped into self-adjoint operators. Furthermore, the map $\hat{A} \mapsto \hat{U} \hat{A} \hat{U}^{-1}$ is continuous in the weak-operator topology, and hence, if $\left\{\hat{A}_{i}\right\}_{i \in I}$ is a weakly-convergent net of operators in $V$, then $\left\{\hat{U} \hat{A}_{i} \hat{U}^{-1}\right\}_{i \in I}$ is a weakly-convergent net of operators in $\ell_{\hat{U}}(V)$, and vice versa.

It follows that $\ell_{\hat{U}}(V)$ is an abelian von Neumann algebra (i.e., it is weakly closed), and hence $\ell_{\hat{U}}$ can be viewed as a map $\ell_{\hat{U}}: \operatorname{Ob}(\mathcal{V}(\mathcal{H})) \rightarrow \operatorname{Ob}(\mathcal{V}(\mathcal{H}))$. We note the following:

1. Clearly, for all $\hat{U}_{1}, \hat{U}_{2} \in \mathcal{U}(\mathcal{H})$,

$$
\ell_{\hat{U}_{1}} \circ \ell_{\hat{U}_{2}}=\ell_{\hat{U}_{1} \hat{U}_{2}}
$$

Thus $\hat{U} \mapsto \ell_{\hat{U}}$ is a realisation of the group $\mathcal{U}(\mathcal{H})$ as a group of transformations of $\mathrm{Ob}(\mathcal{V}(\mathcal{H}))$.

2. For all $U \in \mathcal{U}(\mathcal{H}), V$ and $\ell_{\hat{U}}(V)$ are isomorphic subalgebras of $\mathcal{B}(\mathcal{H})$, and $\ell_{\hat{U}}^{-1}=$ $\ell_{\hat{U}^{-1}}$.

3. If $V^{\prime} \subseteq V$, then, for all $\hat{U} \in \mathcal{U}(\mathcal{H})$,

$$
\ell_{\hat{U}}\left(V^{\prime}\right) \subseteq \ell_{\hat{U}}(V)
$$

Hence, each transformation $\ell_{\hat{U}}$ preserves the partial-ordering of the poset category $\mathcal{V}(\mathcal{H})$

From this it follows that each $\ell_{\hat{U}}: \operatorname{Ob}(\mathcal{V}(\mathcal{H})) \rightarrow \mathrm{Ob}(\mathcal{V}(\mathcal{H}))$ is a functor from the category $\mathcal{V}(\mathcal{H})$ to itself.

4. One consequence of the order-preserving property of $\ell_{\hat{U}}$ is as follows. Let $S$ be a sieve of arrows on $V$, i.e., a collection of subalgebras of $V$ with the property that if $V^{\prime} \in S$, then, for all $V^{\prime \prime} \subseteq V^{\prime}$ we have $V^{\prime \prime} \in S$. Then

$$
\ell_{\hat{U}}(S):=\left\{\ell_{\hat{U}}\left(V^{\prime}\right) \mid V^{\prime} \in S\right\}
$$

is a sieve of arrows on $\ell_{\hat{U}}(V) 25$

\footnotetext{
${ }^{25}$ In the partially ordered set $\mathcal{V}(\mathcal{H})$, an arrow from $V^{\prime}$ to $V$ can be identified with the subalgebra $V^{\prime} \subseteq V$, since there is exactly one arrow from $V^{\prime}$ to $V$.
} 


\subsubsection{The Effect of $\ell_{\hat{U}}$ on Daseinisation}

We recall that if $\hat{P}$ is any projection, then the (outer) daseinisation, $\delta^{o}(\hat{P})_{V}$, of $\hat{P}$ at stage $V$ is

$$
\delta^{o}(\hat{P})_{V}:=\bigwedge\{\hat{Q} \in \mathcal{P}(V) \mid \hat{Q} \succeq \hat{P}\},
$$

where we have resorted once more to using the propositional language $\mathcal{P} \mathcal{L}(S)$. Thus

$$
\begin{aligned}
\hat{U} \delta^{o}(\hat{P})_{V} \hat{U}^{-1} & =\hat{U} \bigwedge\{\hat{Q} \in \mathcal{P}(V) \mid \hat{Q} \succeq \hat{P}\} \hat{U}^{-1} \\
& =\bigwedge\left\{\hat{U} \hat{Q} \hat{U}^{-1} \in \mathcal{P}\left(\ell_{\hat{U}}(V)\right) \mid \hat{Q} \succeq \hat{P}\right\} \\
& =\bigwedge\left\{\hat{U} \hat{Q} \hat{U}^{-1} \in \mathcal{P}\left(\ell_{\hat{U}}(V)\right) \mid \hat{U} \hat{Q} \hat{U}^{-1} \succeq \hat{U} \hat{P} \hat{U}^{-1}\right\} \\
& =\delta^{o}\left(\hat{U} \hat{P} \hat{U}^{-1}\right)_{\ell_{\hat{U}}(V)}
\end{aligned}
$$

where we used the fact that the map $\hat{Q} \mapsto \hat{U} \hat{Q} \hat{U}^{-1}$ is weakly continuous.

Thus we have the important result

$$
\hat{U} \delta^{o}(\hat{P})_{V} \hat{U}^{-1}=\delta^{o}\left(\hat{U} \hat{P} \hat{U}^{-1}\right)_{\ell_{U}}(V)
$$

for all unitary operators $\hat{U}$, and for all stages $V$. There is an analogous result for inner daseinisation.

Equation (5.16) can be applied to the de Groote presheaf $\underline{\mathbb{Q}}$ to give

$$
\hat{U} \delta^{o}(\hat{A})_{V} \hat{U}^{-1}=\delta^{o}\left(\hat{U} \hat{A} \hat{U}^{-1}\right)_{\ell_{\hat{U}}(V)}
$$

for unitary operators $\hat{U}$, and all stages $V$.

We recall that the truth sub-object, $\mathbb{T}^{|\psi\rangle}$, of the outer presheaf, $\underline{O}$, is defined at each stage $V$ by

$$
\begin{aligned}
\mathbb{T}_{V}^{|\psi\rangle} & :=\left\{\hat{\alpha} \in \underline{O}_{V} \mid \operatorname{Prob}(\hat{\alpha} ;|\psi\rangle)=1\right\} \\
& =\left\{\hat{\alpha} \in \underline{O}_{V} \mid\langle\psi|\hat{\alpha}| \psi\rangle=1\right\}
\end{aligned}
$$

The neo-realist, physical interpretation of $\mathbb{T}^{|\psi\rangle}$ is that the 'truth' of the proposition represented by $\hat{P}$ is

$$
\begin{aligned}
\nu\left(\delta^{o}(\hat{P}) \in \mathbb{T} ;|\psi\rangle\right)_{V}:=\left\{V^{\prime} \subseteq V \mid \delta^{o}(\hat{P})_{V^{\prime}} \in \mathbb{T}_{V^{\prime}}^{|\psi\rangle}\right\} \\
=\left\{V^{\prime} \subseteq V \mid\left\langle\psi\left|\delta^{o}(\hat{P})_{V^{\prime}}\right| \psi\right\rangle=1\right\}
\end{aligned}
$$

for all stages $V$. We then get

$$
\begin{aligned}
& \ell_{\hat{U}}\left(\nu\left(\delta^{o}(\hat{P}) \in \mathbb{T} ;|\psi\rangle\right)_{V} \stackrel{\sqrt[5.20]{=}}{=} \ell_{\hat{U}}\left\{V^{\prime} \subseteq V \mid\left\langle\psi\left|\delta^{o}(\hat{P})_{V^{\prime}}\right| \psi\right\rangle=1\right\}\right. \\
& =\left\{\ell_{\hat{U}}\left(V^{\prime}\right) \subseteq \ell_{\hat{U}}(V) \mid\left\langle\psi\left|\delta^{o}(\hat{P})_{V^{\prime}}\right| \psi\right\rangle=1\right\} \\
& =\left\{\ell_{\hat{U}}\left(V^{\prime}\right) \subseteq \ell_{\hat{U}}(V) \mid\left\langle\psi\left|\hat{U}^{-1} \hat{U} \delta^{o}(\hat{P})_{V^{\prime}} \hat{U}^{-1} \hat{U}\right| \psi\right\rangle=1\right\} \\
& \stackrel{5.16}{=}\left\{\ell_{\hat{U}}\left(V^{\prime}\right) \subseteq \ell_{\hat{U}}(V) \mid\left\langle\psi\left|\hat{U}^{-1} \delta^{o}\left(\hat{U} \hat{P} \hat{U}^{-1}\right)_{\ell_{\hat{U}}(V)} \hat{U}\right| \psi\right\rangle=1\right\} \\
& =\quad \nu\left(\delta^{o}\left(\hat{U} \hat{P} \hat{U}^{-1}\right) \in \mathbb{T} ; \hat{U}|\psi\rangle\right)_{\ell_{\hat{U}}(V)} \text {. }
\end{aligned}
$$


Thus we get the important result

$$
\nu\left(\delta^{o}\left(\hat{U} \hat{P} \hat{U}^{-1}\right) \in \mathbb{T} ; \hat{U}|\psi\rangle\right)_{\ell_{\hat{U}}(V)}=\ell_{\hat{U}}\left(\nu\left(\delta^{o}(\hat{P}) \in \mathbb{T} ;|\psi\rangle\right)_{V}\right)
$$

This can be viewed as the topos analogue of the statement in (5.1) about the invariance of the results of quantum theory under the transformations $|\psi\rangle \mapsto \hat{U}|\psi\rangle, \hat{A} \mapsto \hat{U} \hat{A} \hat{U}^{-1}$.

\subsubsection{The $\hat{U}$-twisted Presheaf}

Let us return once more to the definition (5.10) of the functor $\ell_{\hat{U}}: \mathcal{V}(\mathcal{H}) \rightarrow \mathcal{V}(\mathcal{H})$. As we shall see in the next paper, [4, any such functor induces a 'geometric morphism' from $\operatorname{Sets}^{\mathcal{V}(\mathcal{H})^{\mathrm{op}}}$ to $\operatorname{Sets}^{\mathcal{V}(\mathcal{H})^{\mathrm{op}}}$. The exact definition is not needed here: it suffices to remark that part of this geometric morphism is an arrow $\ell_{\hat{U}}^{*}: \operatorname{Sets}^{\mathcal{V}(\mathcal{H})^{\mathrm{op}}} \rightarrow \operatorname{Sets}^{\mathcal{V}(\mathcal{H})^{\mathrm{op}}}$ defined by

$$
\underline{F} \mapsto \ell_{\hat{U}}^{*} \underline{F}:=\underline{F} \circ \ell_{\hat{U}}
$$

Note that, if $\hat{U}_{1}, \hat{U}_{2} \in \mathcal{U}(\mathcal{H})$ then, for all presheaves $\underline{F}$,

$$
\begin{aligned}
\ell_{\hat{U}_{2}}^{*}\left(\ell_{\hat{U}_{1}}^{*} \underline{F}\right) & =\left(\ell_{\hat{U}_{1}}^{*} \underline{F}\right) \circ \ell_{\hat{U}_{2}}=\left(\underline{F} \circ \ell_{\hat{U}_{1}}\right) \circ \ell_{\hat{U}_{2}} \\
& =\underline{F} \circ\left(\ell_{\hat{U}_{1}} \circ \ell_{\hat{U}_{2}}\right)=\underline{F} \circ \ell_{\hat{U}_{1} \hat{U}_{2}} \\
& =\ell_{\hat{U}_{1}}^{*} \underline{F} .
\end{aligned}
$$

Since this is true for all functors $\underline{F}$ in $\operatorname{Sets}^{\mathcal{V}(\mathcal{H})^{\mathrm{op}}}$, we deduce that

$$
\ell_{\hat{U}_{2}}^{*} \circ \ell_{\hat{U}_{1}}^{*}=\ell_{\hat{U}_{1} \hat{U}_{2}}^{*}
$$

and hence the map $\hat{U} \mapsto \ell_{\hat{U}}^{*}$ is an (anti-)representation of the group $\mathcal{U}(\mathcal{H})$ by arrows in the topos $\operatorname{Sets}^{\mathcal{V}(\mathcal{H})^{\mathrm{op}}}$.

Of particular interest to us are the presheaves $\ell_{\hat{U}}^{*} \underline{\Sigma}$ and $\ell_{U}^{*} k\left(\underline{\mathbb{R}^{\succeq}}\right)$. We denote them by $\underline{\Sigma}^{\hat{U}}$ and $k\left(\underline{\mathbb{R}^{\succeq}}\right)^{\hat{U}}$ respectively and say that they are ' $\hat{U}$-twisted'.

Theorem 5.1 For each $\hat{U} \in \mathcal{U}(\mathcal{H})$, there is a natural isomorphism $\iota: \underline{\Sigma} \rightarrow \underline{\Sigma^{\hat{U}}}$ as given in the following diagram

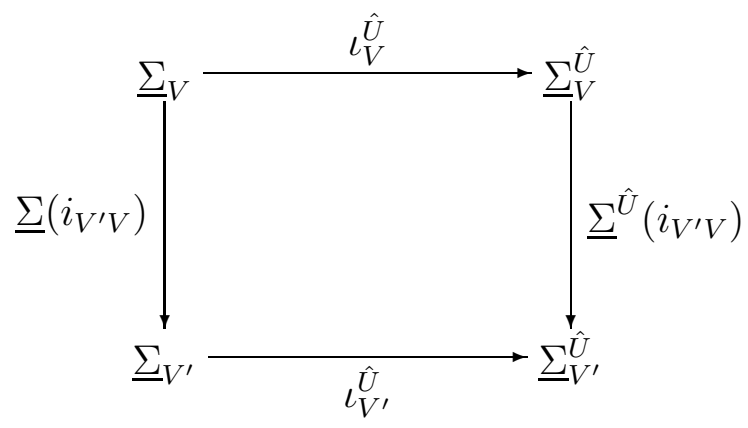


where, at each stage $V$,

$$
\left(\iota_{V}^{U}(\lambda)\right)(\hat{A}):=\lambda\left(\hat{U}^{-1} \hat{A} \hat{U}\right)
$$

for all $\lambda \in \underline{\Sigma}_{V}$, and all $\hat{A} \in V_{\text {sa }}$.

The proof, which just involves chasing round the diagram above using the basic definitions, is not included here.

Even simpler is the following theorem:

Theorem 5.2 For each $\hat{U} \in \mathcal{U}(\mathcal{H})$, there is a natural isomorphism $\kappa^{\hat{U}}: \underline{\mathbb{R}^{\succeq}} \rightarrow\left(\underline{\mathbb{R}^{\succeq}}\right)^{\hat{U}}$ whose components $\kappa_{V}^{\hat{U}}: \underline{\mathbb{R}^{\succeq}} \rightarrow\left(\underline{\mathbb{R}^{\succeq}}\right)_{V}^{\hat{U}}$ are given by

$$
\kappa_{V}^{\hat{U}}(\mu)\left(\ell_{\hat{U}}\left(V^{\prime}\right)\right):=\mu\left(V^{\prime}\right)
$$

for all $V^{\prime} \subseteq V$.

Here, we recall $\mu \in \underline{\mathbb{R}}_{V}$ is a function $\mu: \downarrow V \rightarrow \mathbb{R}$ such that if $V_{2} \subseteq V_{1} \subseteq V$ then $\mu\left(V_{2}\right) \geq \mu\left(V_{1}\right)$, i.e., an order-reversing function. In (5.31) we have used the fact that there is a bijection between the sets $\downarrow_{\hat{U}}(V)$ and $\downarrow V$.

Finally,

Theorem 5.3 We have the following commutative diagram:

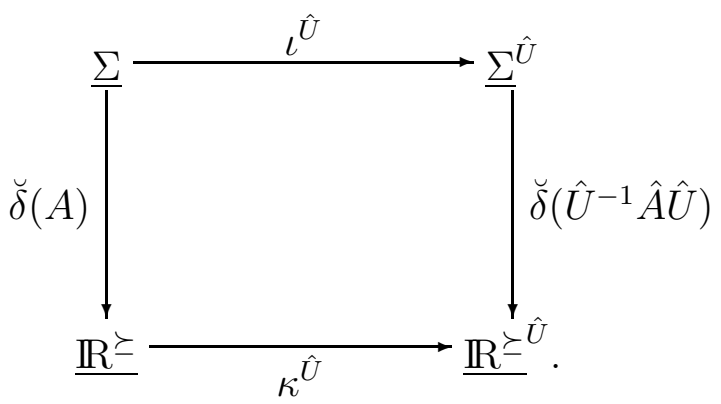

The analogue of unitary operators for a general topos. It is interesting to reflect on the analogue of the above constructions for a general topos. It soon becomes clear that, once again, we encounter the antithetical concepts of 'internal' and 'external'.

For example, in the discussion above, the unitary operators and the group $\mathcal{U}(\mathcal{H})$ lie outside the topos $\operatorname{Sets}^{\mathcal{V}(\mathcal{H})^{\mathrm{op}}}$ and enter directly from the underlying, standard quantum formalism. As such, they are external to both the languages $\mathcal{P} \mathcal{L}(S)$ and $\mathcal{L}(S)$. We anticipate that notions of 'covariance' and 'symmetry' have applications well beyond those in classical physics and quantum physics. However, at the very least, in a general topos one would presumably replace the external $\mathcal{U}(\mathcal{H})$ with an internal group object in the topos concerned. And, of course, the notion of 'symmetry' is closely related to the concept to time, and time development, which opens up a Pandora's box of possible speculation. These issues are important, and await further development. 


\section{Conclusion}

In this, the third in our series of papers on topos theory and physics, we have completed the work begun in the second paper where we showed how propositions can be represented by clopen sub-objects of the spectral presheaf, $\underline{\Sigma}$, of toposified quantum theory. This is equivalent to finding a topos representation of the propositional language $\mathcal{P} \mathcal{L}(S)$, and is the natural completion of the earlier work on quantum theory and topoi.

In the present paper we have gone well beyond this earlier work by finding a representation of the full local language $\mathcal{L}(S)$. In particular, we identified the quantityvalue object as being the presheaf $\underline{\mathbb{R}} \succeq$; or, possibly, its $k$-extension $k(\underline{\mathbb{R}})$ or, $\underline{\mathbb{R}^{\leftrightarrow}}$. This enabled us to find a representation of a physical quantity, $A$, with an arrow $\breve{\delta}^{o}(A): \underline{\Sigma} \rightarrow \underline{\mathbb{R}^{\succeq}}$.

However, we reiterate that our main interest is not to find a new interpretation of quantum theory, but rather to develop a general framework in which new types of theories of physics can be developed; and in which both classical and quantum physics arise as special cases.

It follows that an important challenge for future work is to show that our general topos scheme can be used to develop genuinely new theories of physics, not just to rewrite old ones in a new language. Of particular interest is the problem with which we motivated the scheme in the first place: namely, to find tools for constructing theories that go beyond quantum theory and which do not use Hilbert spaces, path integrals, or any of the other familiar entities in which the continuum real and/or complex numbers play a fundamental role.

As we have discussed, the topoi for quantum systems are of the form $\operatorname{Sets}^{\mathcal{V}(\mathcal{H})^{\mathrm{op}}}$, and hence embody contextual logic in a fundamental way. One way of going 'beyond' quantum theory, while escaping the a priori imposition of continuum concepts, is to use presheaves over a more general 'category of contexts', $\mathcal{C}$, i.e., develop the theory in the topos Sets ${ }^{\mathcal{C}^{\mathrm{p}}}$. Such a structure embodies contextual, multi-valued logic in an intrinsic way, and in that sense might be said to encapsulate one of the fundamental insights of quantum theory. However, and unlike in quantum theory, there is no obligation to use the real or complex numbers in the construction of the category $\mathcal{C}$.

Of course, although true, this remark does not of itself give a concrete example of a theory of this type. However, it is certainly a pointer in a novel direction, and one at which we would not have arrived if the challenge to 'go beyond quantum theory' had been construed only in terms of trying to generalise Hilbert spaces, path integrals, and the like.

From a more general perspective, other types of topoi are possible realms for the construction of physical theories. One simple, but mathematically rich example arises from the theory of $M$-sets. Here, $M$ is a monoid and, like all monoids, can be viewed as a category with a single object, and whose arrows are the elements of $M$. Thought of as a category, a monoid is 'complementary' to a partially-ordered set. In a monoid, 
there is only one object, but plenty of arrows from that object to itself; whereas in a partially-ordered set there are plenty of objects, but at most one arrow between any pair of objects. Thus a partially-ordered set is the most economical category with which to capture the concept of 'contextual logic'. On the other hand, the logic associated with a monoid is non-contextual as there is only one object in the category.

It is easy to see that a functor from $M$ to Sets is just an ' $M$-set': i.e., a set on which $M$ acts as a monoid of transformations. An arrow between two such $M$-sets is an equivariant map between them. In physicists' language, one would say that the topos Sets $^{M}$ — usually denoted $B M$ - is the category of the 'non-linear realisations' of $M$.

The sub-object classifier, $\Omega_{B M}$, in $B M$ is the collection of left ideals in $M$; hence, many of the important constructions in the topos can be handled using the algebraic language. The topos $B M$ is one of the simplest to define and work with and, for that reason, it is a popular source of examples in texts on topos theory. It would be intriguing to experiment with constructing model theories of physics using one of these simple topoi. One possible use of $M$-sets is discussed in [11] in the context of reduction of the state vector, but there will surely be others.

Acknowledgements This research was supported by grant RFP1-06-04 from The Foundational Questions Institute (fqxi.org). AD gratefully acknowledges financial support from the DAAD.

This work is also supported in part by the EC Marie Curie Research and Training Network "ENRAGE" (European Network on Random Geometry) MRTN-CT-2004005616 .

\section{Appendix}

We will show that the mapping

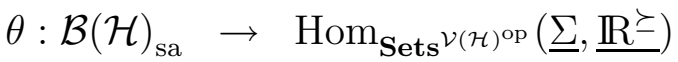

$$
\begin{aligned}
& \hat{A} \mapsto \breve{\delta}^{o}(A)
\end{aligned}
$$

from self-adjoint operators in $\mathcal{B}(\mathcal{H})$ to the natural transformations/arrows from the spectral presheaf $\underline{\Sigma}$ to the quantity-value object $\underline{\mathbb{R}} \succeq$, is injective.

Let $\nu: \underline{\Sigma} \rightarrow \underline{\mathbb{R}^{\succeq}}$ be an arrow in the image of $\theta$. Then there is some self-adjoint operator $\hat{A}$ such that $\nu=\breve{\delta}^{o}(A)$. Our task is to show that $\hat{A}$ is unique. Let $\hat{P} \in \mathcal{P}(\mathcal{H})$, and let $V_{\hat{P}}:=\{\hat{P}, \hat{1}\}^{\prime \prime}$ be the abelian von Neumann algebra generated by $\hat{P}$. Let $\widetilde{H}_{\hat{P}}:=\{\hat{P}, \hat{1}\}$ be the ultrafilter in $\mathcal{P}\left(V_{\hat{P}}\right)$ corresponding to the character $\lambda_{\hat{P}} \in \underline{\Sigma}_{V_{\hat{P}}}$ (i.e., $\left.\lambda_{\hat{P}}(\hat{P})=\lambda_{\hat{P}}(\hat{1})=\hat{1}\right)$. The cone over $\widetilde{H}_{\hat{P}}$ in $\mathcal{P}(\mathcal{H})$ is

$$
\mathcal{C}\left(\widetilde{H}_{\hat{P}}\right)=H_{\hat{P}}=\{\hat{Q} \in \mathcal{P}(\mathcal{H}) \mid \hat{Q} \succeq \hat{P}\}
$$


which is the principal filter in $\mathcal{P}(\mathcal{H})$ generated by $\hat{P} \in \mathcal{P}(\mathcal{H})$. We have

$$
\begin{aligned}
\breve{\delta}^{o}(A)_{V_{\hat{P}}}\left(\lambda_{\hat{P}}\right)\left(V_{\hat{P}}\right) & =\lambda_{\hat{P}}\left(\delta^{o}(\hat{A})_{V_{\hat{P}}}\right) \\
& =f_{\delta^{o}(\hat{A})_{V_{\hat{P}}}}\left(\widetilde{H}_{\hat{P}}\right) \\
& =f_{\hat{A}}\left(\mathcal{C}\left(\widetilde{H}_{\hat{P}}\right)\right) \\
& =f_{\hat{A}}\left(H_{\hat{P}}\right) .
\end{aligned}
$$

Here, we used (3.32) and (3.31). Letting $\hat{P}$ vary over $\mathcal{P}(\mathcal{H})$, we get the values of the observable function $f_{\hat{A}}$ at all principal filters $H_{\hat{P}}$.

De Groote has shown [7] that there is a bijection

$$
\begin{aligned}
\omega: \mathcal{B}(\mathcal{H})_{\mathrm{sa}} & \rightarrow \mathcal{O}(\mathcal{D}(\mathcal{H})) \\
\hat{A} & \mapsto f_{\hat{A}}
\end{aligned}
$$

between self-adjoint operators and observable functions $f_{\hat{A}}: \mathcal{D}(\mathcal{H}) \rightarrow \mathbb{R}$. Moreover, each observable function $f_{\hat{A}}$ is uniquely determined by its restriction to principal filters. This shows that, given an arrow $\nu$ in the image of $\theta$, we can reconstruct from it a unique self-adjoint operator $\hat{A} \in \mathcal{B}(\mathcal{H})_{\text {sa }}$.

Now let

$$
\left[\breve{\delta}^{o}(A)\right]: \underline{\Sigma} \rightarrow k(\underline{\mathbb{R}} \underline{\underline{\mathbb{R}}})
$$

denote the natural transformation from the spectral presheaf to the abelian groupobject $k\left(\underline{\mathbb{R}^{\succeq}}\right)$, given by first sending $\hat{A}$ to $\breve{\delta}^{o}(A)$ and then taking the $k$-equivalence classes at each stage $V$. The monoid $\underline{\mathbb{R}^{\succeq}}$ is embedded into $k\left(\underline{\mathbb{R}^{\succeq}}\right)$ by sending $\mu \in \underline{\mathbb{R}}_{V}^{\succeq}$ to $[\mu, 0] \in k\left(\underline{\mathbb{R}^{\succeq}}\right)_{V}$ for all $V$, which implies that $\hat{A}$ is also uniquely determined by $\left[\breve{\delta}^{o}(A)\right] \underline{26}^{26}$

On the level of the local language $\mathcal{L}(S)$, we will assume that $\mathcal{L}(S)$ contains function symbols $A: \Sigma \rightarrow \mathcal{R}$ corresponding bijectively to a set of self-adjoint operators $\hat{A} \in \mathcal{B}(\mathcal{H})_{\text {sa }}$. Hence, these function symbols are faithfully represented by the natural transformations $\breve{\delta}^{o}(A)$.

Interestingly, these results all carry over to an arbitrary unital von Neumann algebra $\mathcal{N} \subseteq \mathcal{B}(\mathcal{H})$. In this way, the formalism is flexible enough to adapt to situations where we have symmetries (which can described mathematically by a von Neumann algebra $\mathcal{N}$ that has a non-trivial commutant) and super-selection rules (which corresponds to $\mathcal{N}$ having a non-trivial centre).

\section{References}

[1] S. MacLane and I. Moerdijk. Sheaves in Geometry and Logic: A First Introduction to Topos Theory. Springer-Verlag, London (1992).

\footnotetext{
${ }^{26}$ In an analogous manner, one can show that the arrows $\breve{\delta}^{i}(A): \underline{\Sigma} \rightarrow \underline{\mathbb{R}} \underline{\underline{ }}$ and $\left[\breve{\delta}^{i}(A)\right]: \underline{\Sigma} \rightarrow k(\underline{\mathbb{R}} \underline{ })$ uniquely determine $\hat{A}$, and that the arrow $\breve{\delta}(A): \underline{\Sigma} \rightarrow \underline{\mathbb{R} \leftrightarrow}$ also uniquely determines $\hat{A}$. It is currently unknown if the arrow $[\breve{\delta}(A)]: \underline{\Sigma} \rightarrow \underline{\mathbb{R}^{\leftrightarrow}} / \equiv$ also fixes $\hat{A}$ uniquely.
} 
[2] A. Döring and C.J. Isham. A topos foundation for theories of physics: I. Formal languages for physics. (2007).

[3] A. Döring and C.J. Isham. A topos foundation for theories of physics: II. Daseinisation and the liberation of quantum theory. (2007).

[4] A. Döring and C.J. Isham. A topos foundation for theories of physics: IV. Categories of systems. (2007).

[5] H.F. de Groote. On a canonical lattice structure on the effect algebra of a von Neumann algebra. arxiv.org/abs/math-ph/0410018 v2, (2004).

[6] H.F. de Groote. Observables. arxiv.org/abs/math-ph/0507019, (2005).

[7] H.F. de Groote. Observables II: Quantum Observables. arxiv.org/abs/mathph/0509075, (2005).

[8] A. Döring. Kochen-Specker theorem for von Neumann algebras. Int. J. Theor. Phys. 44, 139-160, (2005).

[9] A. Döring. Observables as functions: Antonymous functions. arxiv.org/abs/quantph/0510102, (2005).

[10] A. Döring. Daseinisation and functions on dual ideals. In preparation, (2007).

[11] C.J. Isham. A Topos Perspective on State-Vector Reduction. Int. J. Theor. Phys. To appear, (2007).

[12] C.J. Isham and J. Butterfield. A topos perspective on the Kochen-Specker theorem: I. Quantum states as generalised valuations. Int. J. Theor. Phys. 37, 26692733, (1998).

[13] C.J. Isham and J. Butterfield. A topos perspective on the Kochen-Specker theorem: II. Conceptual aspects, and classical analogues. Int. J. Theor. Phys. 38, 827-859, (1999).

[14] J. Hamilton, J. Butterfield and C.J. Isham. A topos perspective on the KochenSpecker theorem: III. Von Neumann algebras as the base category. Int. J. Theor. Phys. 39, 1413-1436, (2000).

[15] J. Butterfield and C.J. Isham A topos perspective on the Kochen-Specker theorem: IV. Interval valuations. Int. Jour. Theor. Phys. 41, 613-639, (2002).

[16] M. Jackson. A sheaf theoretic approach to measure theory. PhD thesis, University of Pittsburgh, (2006).

[17] M.P. Olson. The Selfadjoint Operators of a von Neumann Algebra Form a Conditionally Complete Lattice. Proc. of the AMS 28, 537-544, (1971).

[18] A.C. Grayling. An Introduction to Philosophical Logic. Duckworth Press, London (1990). 